\title{
A FATOU THEOREM \\ FOR THE SOLUTION OF THE HEAT EQUATION AT THE CORNER POINTS OF A CYLINDER
}

\author{
KIN MING HUI
}

\begin{abstract}
In this paper the author proves existence and uniqueness of the initial-Dirichlet problem for the heat equation in a cylindrical domain $D \times$ $(0, \infty)$ where $D$ is a bounded smooth domain in $R^{n}$ with zero lateral values. A unique representation of the strong solution is given in terms of measures $\mu$ on $D$ and $\lambda$ on $\partial D$. We also show that the strong solution $u(x, t)$ of the heat equation in a cylinder converges a.e. $x_{0} \in \partial D \times\{0\}$ as $(x, t)$ converges to points on $\partial D \times\{0\}$ along certain nontangential paths.
\end{abstract}

\section{INTRODUCTION}

The existence and uniqueness of the initial-Dirichlet problem for the heat equation in a cylindrical domain $D \times(0, T)$ subject to Dirichlet boundary conditions $\left.u\right|_{\partial D \times(0, T)} \equiv 0$ where $D$ is a bounded smooth domain in $R^{n}$ have been studied by a large number of researchers. (See [F, LSU, FGS].)

In this paper, by following the argument of Dahlberg and Kenig [DK2], I prove the existence and uniqueness of the nonnegative strong solution of the initial Dirichlet problem (IDP) for the heat equation in a cylinder $D \times(0, \infty)$, $D \in C^{\infty}$, with Dirichlet boundary condition $u_{\partial D \times(0, \infty)} \equiv 0$.

In fact I show that corresponding to each nonnegative strong solution $u(x, t)$ of IDP, there exists a pair of measures $\mu$ on $D$ and $\lambda$ on $\partial D$ such that

$$
\begin{aligned}
u(x, t)= & \int_{D} G(X, t ; Q, 0) d \mu(Q) \\
& +\int_{\partial D} \frac{\partial G}{\partial N_{Q}}(x, t ; Q, 0) d \lambda(Q)
\end{aligned}
$$

where $G(x, t ; Q, s)$ is the Green function for the heat equation and $\partial / \partial N_{Q}$ is the derivative in the direction of the inward normal at $Q$.

I also find that the strong solution $u(x, t)$ of the heat equation in a cylinder converges a.e. $x_{0} \in \partial D \times\{0\}$ as $(x, t)$ converges to points on $\partial D \times\{0\}$ along certain nontangential path. In fact I prove that

$$
\lim _{(x, t) \in \bar{\Gamma}_{\beta}\left(x_{0}\right)} u(x, t)=\frac{\beta}{\sqrt{4 \pi}} \cdot \frac{d \lambda}{d \sigma} \quad \text { a.e. } x_{0} \in \partial D \times\{0\}
$$

Received by the editors June 6, 1990.

1980 Mathematics Subject Classification (1985 Revision). Primary 35K05, 35K15, 35K20, 35C15, 35D05; Secondary 31B10, 31B25.

Key words and phrases. Heat equation, initial-Dirichlet problem, Fatou theorem at corner points. 
where $\bar{\Gamma}_{\beta}\left(x_{0}\right)=\left\{\left(x^{\prime}, x_{n}, t\right) \in D \times(0, T):\left|x^{\prime}\right| \leq C x_{n}, x_{n}=\beta t^{3 / 2}\right\}, x=$ $\left(x^{\prime}, x_{n}\right)$ is the local coordinate of the point $x$ with respect to the local coordinate system at $x_{0}$ with origin at $x_{0}$ and with the plane $\left\{x_{n}=0\right\}$ tangent to $\partial D$ at $x_{0}$, and $\frac{d \lambda}{d \sigma}$ is the Radon-Nikodym derivative of $d \lambda$ with respect to the surface measure $d \sigma$ on $\partial D$.

While the existence of Fatou type limit on the lateral surface and bottom of a cylinder has been investigated by Fabes, Garofalo, and Salsa [FGS], Fabes and Salsa [FS] in the case of nondegenerate parabolic equation, and by Kemper $[\mathrm{K}]$ in the case of heat equation, nothing is known about the behaviour of the solution $u(x, t)$ as $(x, t)$ tends to $\partial D \times\{0\}$. My result is entirely new. I have also shown that the index $\frac{3}{2}$ appearing in the definition of $\bar{\Gamma}_{\beta}\left(x_{0}\right)$ is essentially sharp.

By using the argument of $\mathrm{M}$. Grüter and K. O. Widman [GW], we will establish various estimates for the Green's function of the heat equation in $D \times(0, \infty)$ in $\S 1$. (The author was informed by Professor Russell Brown that similar estimates were obtained by E. B. Davies [D] using logarithmic Sobolev inequalities.) In subsection 2.1 , we will show that any strong solution $u$ of the (IDP) has a trace $\mu$ on $D$ and a trace $\lambda$ on $\partial D$ with $\int_{x \in D} \delta(x) d \mu(x)<\infty$, $\int_{Q \in \partial D} d \lambda(Q)<\infty, \delta(x)=\operatorname{dist}(x, \partial D)$ and $\forall \eta \in C^{\infty}\left(R^{n}\right),\left.\eta\right|_{\partial D} \equiv 0$,

$$
\lim _{t \rightarrow 0} \int_{D} u(x, t) \eta(x) d x=\int_{D} \eta d \mu+\int_{\partial D} \frac{\partial \eta}{\partial N} d \lambda
$$

following the same line of proof as [DK2].

In subsection 2.3 , we will prove some priori estimates for the strong solutions $u$ of the (IDP) of the heat equation in a cylinder. In subsection 2.4, we will use the methods in $[\mathrm{JK}],[\mathrm{K}]$ and [FGS] to prove the convergence a.e. on $\partial D \times\{0\}$ of $u(x, t)$ as $(x, t) \rightarrow\left(Q_{0}, 0\right) \in \partial D \times\{0\}$ along the nontangential paths $\Gamma_{\beta}\left(Q_{0}\right)$ by assuming the everywhere convergence a.e. of the solution $\bar{u}$ of the heat equation at the corner points $\partial D \times\{0\}$ with initial trace $(d \mu, d \lambda)=(0, d \sigma)$ where $d \sigma$ is the surface measure on $\partial D$ along these nontangential paths.

And in subsection 2.5 , we will finish the proof by proving the everywhere convergence of such solution $\bar{u}$ along

$$
\begin{aligned}
\bar{\Gamma}_{\beta}\left(Q_{0}\right)=\left\{(Q, s)=\left(Q^{\prime}, Q_{n}, s\right) \in\right. & R^{n-1} \times R^{+} \times R^{-}: \\
& \left.\left|Q^{\prime}\right| \leq M Q_{n}, Q_{n}=\beta(-s)^{3 / 2} \leq \alpha\right\}
\end{aligned}
$$

for all corner points $\left(Q_{0}, 0\right) \in \partial D \times\{0\}$ by using layer potential method. Finally in subsection 2.6 we will show that the index $\frac{3}{2}$ on $t$ in the definition of the nontangential cones at corner points are essentially sharp.

\section{Estimates FOR THE GREEN FUNCTION}

In this section we will establish various estimates on $G(x, t ; Q, s)$ the Green kernel of the heat equation in $D \times(0, \infty)$ following basically the line of proofs of [GW] for the estimates on the Green's function for the Laplacian. We will start with a lemma.

Lemma 1.1. Let $u_{R}$ be a solution of the heat equation in $D_{R} \times\left(0,(2 R)^{2}\right)$ where $D_{R}=B(0,2 R) \backslash \overline{B(0, R)}$ with boundary value given by $\phi\left(x / 2 R, t /(2 R)^{2}\right)$ where 
$\phi \in C^{\infty}\left(\partial_{p}\left(D_{1} \times(0, \infty)\right)\right)$ with

$$
\begin{cases}\phi(x, t) \equiv 0 & \text { for }(x, t) \in \partial B(0,1) \times[0,1) \cup(B(0,1) \backslash B(0,1 / 2)) \times\{0\}, \\ \phi(x, t) \equiv 0 & \text { for }(x, t) \in \partial B(0,1 / 2) \times(1 / 2,1),\end{cases}
$$

and $0 \leq \phi \leq 1$. Then

$$
\left\|\nabla_{x} u_{R}(x, t)\right\|_{L^{\infty}\left(D_{R} \times\left(0,(2 R)^{2}\right)\right)} \leq \frac{C}{R}<\infty
$$

for some constant $C>0$ independent of $R$.

Proof. The lemma follows from the boundary Schauder's estimate. (See [F, p. 65, Theorem 6].)

Corollary 1.2. Let $v_{R}$ be a solution of the backward heat equation $\partial_{s} v_{R}+\Delta v_{R}=0$ in $D_{R} \times\left(-(2 R)^{2}, 0\right)$ where $D_{R}=B(0,2 R) \backslash \overline{B(0, R)}$ with boundary value $\phi$ where $\phi \in C^{\infty}$ and

$$
\begin{cases}\phi(Q, s) \equiv 1 & \text { for }(Q, s) \in \partial B(0,2 R) \times\left(-(2 R)^{2}, 0\right] \\ & \text { or }(Q, s) \in(B(0,2 R) \backslash \overline{B(0, R)}) \times\{0\}, \\ \phi(Q, s) \equiv 0 & \text { for }(Q, s) \in \partial B(0, R) \times\left(-(2 R)^{2},-2 R^{2}\right)\end{cases}
$$

and $0 \leq \phi \leq 1$. Then

$$
\left\|\nabla_{x} v_{R}(x, t)\right\|_{L^{\infty}\left(D_{R} \times\left(-(2 R)^{2}, 0\right)\right)} \leq \frac{C}{R}<\infty
$$

for some constant $C>0$ independent of $R$.

Lemma 1.3. For $0 \leq s<t \leq T, T>0$, we have

(i)

$$
G(P, t ; Q, s) \leq \frac{C}{(t-s)^{n / 2}} e^{-c|P-Q|^{2} /(t-s)}, \quad P, Q \in D
$$

(ii)

$$
G(P, t ; Q, s) \leq \frac{C \delta(P)}{(t-s)^{n+1 / 2}} e^{-c|P-Q|^{2} /(t-s)}, \quad P, Q \in D
$$

(iii)

$$
G(P, t ; Q, s) \leq \frac{C \delta(P) \delta(Q)}{(t-s)^{n+2 / 2}} e^{-c|P-Q|^{2} /(t-s)}, \quad P, Q \in D
$$

$$
\left|\frac{\partial G}{\partial N_{Q}}(P, t ; Q, s)\right| \leq \frac{C \delta(P)}{(t-s)^{n+2 / 2}} e^{-c|P-Q|^{2} /(t-s)}, \quad P \in D, Q \in \partial D,
$$

where $\partial / \partial N_{Q}$ is the derivative in the direction of the normal to $\partial D$ at the point $Q \in \partial D, \delta(P)=\operatorname{dist}(P, \partial D)$, and $C$ is a constant independent of $P, Q, t, s$.

Proof. (i) is proved in [LSU]. To prove (ii), note that since $D$ is smooth, $\partial D$ satisfies the exterior sphere condition, i.e. there exists a positive constant $h>0$ such that for each $x \in \partial D, \forall 0<r \leq h$, there exists $x_{0} \in D^{c}$ such that $\overline{B\left(x_{0}, r\right)} \cap \bar{D}=\{x\}$.

Case 1. $\delta(P) \geq h$. 
Then

So by (i)

$$
1 \leq \frac{\delta(P)}{h} \leq \frac{\delta(P)}{h} \cdot \frac{(t-s)^{1 / 2}}{(t-s)^{1 / 2}} \leq C \frac{\delta(P)}{(t-s)^{1 / 2}} .
$$

$$
\begin{aligned}
G(P, t ; Q, s) & \leq \frac{C^{\prime}}{(t-s)^{n / 2}} e^{-c|P-Q|^{2} /(t-s)} \cdot \frac{\delta(P)}{(t-s)^{1 / 2}} \\
& \leq C^{\prime} \frac{\delta(P)}{(t-s)^{n+1 / 2}} e^{-c|P-Q|^{2} /(t-s)} .
\end{aligned}
$$

Case 2. $\delta(P) \geq(t-s)^{1 / 2} / 4$.

Then $1 / 4 \leq \delta(P) /(t-s)^{1 / 2}$. So by $(\mathrm{i})$

$$
\begin{aligned}
G(P, t ; Q, s) & \leq \frac{C}{(t-s)^{n / 2}} e^{-c|P-Q|^{2} /(t-s)} \cdot \frac{4 \delta(P)}{(t-s)^{1 / 2}} \\
& \leq C^{\prime} \frac{\delta(P)}{(t-s)^{n+1 / 2}} e^{-c|P-Q|^{2} /(t-s)}
\end{aligned}
$$

Case 3. $\delta(P) \leq h$ and $\delta(P) \leq(t-s)^{1 / 2} / 4 \leq h$.

We fix $P^{*} \in \partial D$ with $\left|P-P^{*}\right|=\delta(P)$ and set $R=(t-s)^{1 / 2} / 4$. Since $D$ satisfies the exterior sphere condition, there exists a $P_{0} \in D^{c}$ such that $\overline{B\left(P_{0}, R\right)} \cap \bar{D}=\left\{P^{*}\right\}$. We may also assume without loss of generality that $\overline{P P^{*}} \subset D$.

Consider the cylinder $Q_{R}^{\prime}=D_{R}^{\prime} \times\left(t-(2 R)^{2}, t\right], D_{R}^{\prime}=B\left(P_{0}, 2 R\right) \backslash \overline{B\left(P_{0}, R\right)}$. Then $(P, t) \in \overline{Q_{R}^{\prime}} \cap D \times(0, \infty)$ and for any $\left(P^{\prime}, t^{\prime}\right) \in \overline{Q_{R}^{\prime}} \cap \bar{D} \times(0, \infty)$,

$$
\begin{aligned}
t-s & \geq t^{\prime}-s \geq t-(2 R)^{2}-s \\
& \geq t-s-\left(\frac{(t-s)^{1 / 2}}{2}\right)^{2}=\frac{3(t-s)}{4} .
\end{aligned}
$$

Now we have either

$$
|t-s|^{1 / 2} \leq \frac{|P-Q|}{2}
$$

or

$$
|t-s|^{1 / 2} \geq \frac{|P-Q|}{2} .
$$

If (a) holds, then $R \leq|P-Q| / 8$, so

$$
\begin{aligned}
\left|P^{\prime}-Q\right| & \geq|P-Q|-\left|P-P^{\prime}\right| \\
& \geq|P-Q|-\left(\left|P-P_{0}\right|+\left|P_{0}-P^{\prime}\right|\right) \\
& \geq|P-Q|-(2 R+2 R) \\
& \geq \frac{|P-Q|}{2} .
\end{aligned}
$$

Therefore

$$
\begin{aligned}
G\left(P^{\prime}, t^{\prime} ; Q, s\right) & \leq \frac{C}{\left(t^{\prime}-s\right)^{n / 2}} e^{-c\left|P^{\prime}-Q\right|^{2} /\left(t^{\prime}-s\right)} \quad \text { by (i) } \\
& \leq \frac{C^{\prime}}{(t-s)^{n / 2}} e^{-c|P-Q|^{2} /(t-s)}
\end{aligned}
$$


If (b) holds, then $|P-Q|^{2} /(t-s) \leq 4 \Rightarrow e^{-c|P-Q|^{2} /(t-s)} \geq e^{-4 C^{\prime}}$. Therefore

$$
\begin{aligned}
G\left(P^{\prime}, t^{\prime} ; Q, s\right) & \leq \frac{C}{\left(t^{\prime}-s\right)^{n / 2}} e^{-c\left|P^{\prime}-Q\right|^{2} /\left(t^{\prime}-s\right)} \quad \text { by }(\mathrm{i}) \\
& \leq \frac{C}{\left(t^{\prime}-s\right)^{n / 2}} \leq \frac{C^{\prime \prime}}{(t-s)^{n / 2}} e^{-c^{\prime}|P-Q|^{2} /(t-s)}
\end{aligned}
$$

In both cases, we have

$$
G\left(P^{\prime}, t^{\prime} ; Q, s\right) \leq C \frac{1}{(t-s)^{n / 2}} e^{-c^{\prime}|P-Q|^{2} /(t-s)}
$$

If $\bar{u}_{R}\left(P^{\prime}, t^{\prime}\right)=u_{R}\left(P^{\prime}-P_{0}, t^{\prime}-\left(t-(2 R)^{2}\right)\right),\left(P^{\prime}, t^{\prime}\right) \in \overline{Q_{R}^{\prime}}$ where $u_{R}$ is as in Corollary 5.2, then since

$$
\left\{\begin{aligned}
G\left(P^{\prime}, t^{\prime} ; Q, s\right) & =0 \\
\bar{u}_{R}\left(P^{\prime}, t^{\prime}\right) & \geq 0, \quad \forall\left(P^{\prime}, t^{\prime}\right) \in \partial D \times\left[t-(2 R)^{2}, t\right) \cap \overline{Q_{R}^{\prime}}
\end{aligned}\right.
$$

and

$$
\left\{\begin{aligned}
G\left(P^{\prime}, t^{\prime} ; Q, s\right) & \leq C \frac{1}{(t-s)^{n / 2}} e^{-c^{\prime}|P-Q|^{2} /(t-s)}, \\
\bar{u}_{R}\left(P^{\prime}, t^{\prime}\right) & \equiv 1, \quad \forall\left(P^{\prime}, t^{\prime}\right) \in \partial_{p} Q_{R}^{\prime} \cap D \times(0, \infty),
\end{aligned}\right.
$$

by applying the maximum principle to the functions

$$
G(\cdot, \cdot ; Q, s) \text { and } C \frac{1}{(t-s)^{n / 2}} e^{-c^{\prime}|P-Q|^{2} /(t-s)} \bar{u}_{R}(\cdot, \cdot)
$$

in the region $Q_{R} \cap D \times(0, \infty)$ we have:

$$
G(P, t ; Q, s) \leq C \frac{1}{(t-s)^{n / 2}} e^{-c|P-Q|^{2} /(t-s)} \bar{u}_{R}(P, t)
$$

since $(P, t) \in \overline{Q_{R}^{\prime}} \cap D \times(0, \infty)$.

$$
\leq C \frac{\delta(P)}{(t-s)^{n / 2}} e^{-c|P-Q|^{2} /(t-s)} \cdot\left|\frac{\partial \bar{u}_{R}}{\partial l_{P^{*}}}(\bar{P}, t)\right|
$$

where $\partial / \partial l_{P^{*}}$ is the derivative in the direction $\overline{P P^{*}}$ and $\bar{P}$ is some point on $\overline{P P^{*}}$.

$$
\begin{aligned}
& \leq C \frac{\delta(P)}{(t-s)^{n / 2}} e^{-c|P-Q|^{2} /(t-s)} \cdot \frac{C^{\prime}}{(t-s)^{1 / 2}} \quad(\text { by Corollary 1.2) } \\
& \leq C^{\prime} \frac{\delta(P)}{(t-s)^{n+1 / 2}} e^{-c|P-Q|^{2} /(t-s)}
\end{aligned}
$$

Case 4. $\delta(P) \leq h$ and $\delta(P) \leq h \leq 4 h \leq(t-s)^{1 / 2}$. 
We fix $P^{*} \in \partial D$ as before and set $R=h$. Since now $R=h \leq(t-s)^{1 / 2} / 4$, the same arguments as in Case 3 imply

$$
\begin{aligned}
G(P, t ; Q, s) & \leq C \frac{1}{(t-s)^{n / 2}} e^{-c|P-Q|^{2} /(t-s)} \bar{u}_{R}(P, t) \\
& \leq C \frac{\delta(P)}{(t-s)^{n / 2}} e^{-c|P-Q|^{2} /(t-s)} \\
& \leq C_{T} \frac{\delta(P)}{(t-s)^{n+1 / 2}} e^{-c|P-Q|^{2} /(t-s)} \quad \text { since } 0 \leq s<t \leq T .
\end{aligned}
$$

Proof of (iii). The proof of (iii) is analogous to the proof of (ii).

(iv) follows from (iii) by dividing both sides of (iii) by $\delta(Q)$ and then letting $Q \rightarrow Q_{0} \in \partial D$ along the normal to $\partial D$ at $Q_{0}$.

Corollary 1.4. The following is true:

$$
\begin{gathered}
\left|\nabla_{Q} G(P, t ; Q, s)\right| \leq C \frac{\delta(P)}{(t-s)^{n+2 / 2}} e^{-c|P-Q|^{2} /(t-s)}, \\
\left|\nabla_{P} G(P, t ; Q, s)\right| \leq C \frac{\delta(Q)}{(t-s)^{n+2 / 2}} e^{-c|P-Q|^{2} /(t-s)}, \\
\left|\nabla_{Q} \nabla_{P} G(P, t ; Q, s)\right| \leq C \frac{1}{(t-s)^{n+2 / 2}} e^{-c|P-Q|^{2} /(t-s)},
\end{gathered}
$$

for all $0 \leq s<t \leq T$ (for any fixed $T>0$ ), $P, Q \in D$, and $C$ is a constant depending only on $T>0$.

Proof. Case 1. $\delta(Q) \leq(t-s)^{1 / 2}$.

Apply Schauder interior estimate to the function $G(P, t, \cdot, \cdot)$ in the cylin$\operatorname{der} Q_{R}^{\prime}=B(Q, R) \times\left(s, s+R^{2}\right)$ with $R=\delta(Q) / 2$. We get $\forall i=1, \ldots, n$,

$$
\begin{aligned}
\left|\frac{\partial G}{\partial Q_{i}}(P, t ; Q, s)\right| \cdot \frac{\delta(Q)}{2} & \leq C \sup _{\left(Q^{\prime}, s^{\prime}\right) \in Q_{R}^{\prime}} G\left(P, t ; Q^{\prime}, s^{\prime}\right) \\
& \leq C \sup _{\left(Q^{\prime}, s^{\prime}\right) \in Q_{R}^{\prime}} \frac{\delta(P) \delta\left(Q^{\prime}\right)}{\left(t-s^{\prime}\right)^{n+2 / 2}} e^{-c\left|P-Q^{\prime}\right|^{2} /\left(t-s^{\prime}\right)} .
\end{aligned}
$$

Now for any $\left(Q^{\prime}, s^{\prime}\right) \in Q_{R}^{\prime}$,

$$
\begin{aligned}
s \leq s^{\prime} & \leq s+R^{2} \\
\Rightarrow t-s & \geq t-s^{\prime} \geq t-s-R^{2} \\
& =t-s-\left(\frac{\delta(Q)}{2}\right)^{2} \geq t-s-\left(\frac{(t-s)^{1 / 2}}{2}\right)^{2} \\
& \geq \frac{3(t-s)}{4} .
\end{aligned}
$$

Let $Q^{*}$ be a point on $\partial D$ such that $\left|Q-Q^{*}\right|=\delta(Q)$. Then

$$
\begin{aligned}
\delta\left(Q^{\prime}\right) & \leq\left|Q^{\prime}-Q^{*}\right| \leq\left|Q^{\prime}-Q\right|+\left|Q-Q^{*}\right| \\
& \leq \frac{1}{2} \delta(Q)+\delta(Q)=\frac{3 \delta(Q)}{2} .
\end{aligned}
$$


Also we have either

$$
(t-s)^{1 / 2} \leq \frac{|P-Q|}{2}
$$

or

$$
(t-s)^{1 / 2}>\frac{|P-Q|}{2}
$$

If (a) holds, then

$$
\begin{aligned}
\left|P-Q^{\prime}\right| & \geq|P-Q|-\left|Q-Q^{\prime}\right| \geq|P-Q|-R \\
& \geq|P-Q|-\left(\frac{\delta(Q)}{2}\right) \geq|P-Q|-\frac{(t-s)^{1 / 2}}{2} \\
& \geq|P-Q|-\frac{|P-Q|}{4}=\frac{3|P-Q|}{4} .
\end{aligned}
$$

So

$$
e^{-c\left|P-Q^{\prime}\right|^{2} /\left(t-s^{\prime}\right)} \leq e^{-c^{\prime}|P-Q|^{2} /(t-s)}
$$

If (b) holds, then

$$
|P-Q|^{2} /(t-s) \leq 4 \Rightarrow e^{-c^{\prime}|P-Q|^{2} /(t-s)} \geq e^{-4 C^{\prime}} \geq e^{-c^{\prime}\left|P-Q^{\prime}\right|^{2} /\left(t-s^{\prime}\right)}
$$

since $e^{-c^{\prime}\left|P-Q^{\prime}\right|^{2} /\left(t-s^{\prime}\right)} \leq 1$. In both cases we have

$$
e^{-c^{\prime}\left|P-Q^{\prime}\right|^{2} /\left(t-s^{\prime}\right)} \leq C e^{-c^{\prime}|P-Q|^{2} /(t-s)} .
$$

Combining the above inequalities we have

$$
\sup _{\left(Q^{\prime}, s^{\prime}\right) \in Q_{R}^{\prime}} \frac{\delta(P) \delta\left(Q^{\prime}\right)}{\left(t-s^{\prime}\right)^{n+2 / 2}} e^{-c\left|P-Q^{\prime}\right|^{2} /\left(t-s^{\prime}\right)} \leq C \frac{\delta(P) \delta(Q)}{(t-s)^{n+2 / 2}} e^{-c|P-Q|^{2} /(t-s)} .
$$

Hence

$$
\begin{aligned}
\left|\frac{\partial G}{\partial Q_{i}}(P, t ; Q, s)\right| \cdot \frac{\delta(Q)}{2} & \leq C \frac{\delta(P) \delta(Q)}{(t-s)^{n+2 / 2}} e^{-c|P-Q|^{2} /(t-s)} \\
\Rightarrow\left|\nabla_{Q} G(P, t ; Q, s)\right| & \leq C \frac{\delta(P)}{(t-s)^{n+2 / 2}} e^{-c|P-Q|^{2} /(t-s)} .
\end{aligned}
$$

Case 2. $\delta(Q) \geq(t-s)^{1 / 2}$. The proof is the same as in Case 1 except that we apply the Schauder interior estimate to $G(P, t ; \cdot, \cdot)$ in $Q_{R}^{\prime}=B(Q, R) \times$ $\left(s, s+R^{2}\right)$ with $R=(t-s)^{1 / 2} / 2$, and use (ii) of Lemma 1.3 to control $G\left(P, t ; Q^{\prime}, S^{\prime}\right),\left(Q^{\prime}, s^{\prime}\right) \in Q_{R}^{\prime}$.

The proof of (ii) and (iii) is similar to the proof of (i). We omit the details.

\section{THE HEAT EQUATION IN A CYLINDER}

2.1. The initial Dirichlet problem. In this subsection we will follow the argument in [DK2] to prove that all strong solutions $u(x, t)$ are in one-to-one correspondence with suitable pairs of measures $\mu$ on $D$ and $\lambda$ on $\partial D$. We will also show that any strong solution $u$ has an explicit representation given by

$$
u(x, t)=\int_{Q \in D} G(x, t ; Q, 0) d \mu(Q)+\int_{Q \in \partial D} \frac{\partial G}{\partial N_{Q}}(x, t ; Q, 0) d \lambda(Q)
$$


where $\partial / \partial N_{Q}$ is the derivative in the direction of the inward normal $N_{Q}$ at $Q \in \partial D$.

We will start with some definitions and results of [DK2]. We say that $u$ is a strong solution of the initial Dirichlet problem (IDP) for the equation $\Delta u=\partial_{t} u$ in $D \times(0, \infty)$ if $u$ is a continuous, nonnegative function in $\bar{D} \times(0, \infty), u \equiv 0$ on $\partial D \times(0, \infty)$ and for all smooth functions $\eta$ on $\bar{D} \times(0, \infty)$ which vanish on $\partial D \times\left[\tau_{1}, \tau_{2}\right], \tau_{1}>0$, we have

$$
\begin{aligned}
\iint_{D \times\left[\tau_{1}, \tau_{2}\right]}\left[u \Delta \eta+u \frac{\partial \eta}{\partial t}\right] d x d t & \\
= & \int_{D} u\left(x, \tau_{2}\right) \eta\left(x, \tau_{2}\right) d x-\int_{D} u\left(x, \tau_{1}\right) \eta\left(x, \tau_{1}\right) d x .
\end{aligned}
$$

Lemma 2.1.1 [DK2, Lemma 2]. Let $u_{1}, u_{2}$ be strong solutions of the (IDP) in $D \times(0, \infty)$. Let $\eta \geq 0, \eta \in C_{0}^{\infty}(D)$, and let $T<\infty,\left\{\tau_{j}\right\} \searrow 0, \tau_{0}=T$. Then there exist nonnegative measures $\left\{\lambda_{j}\right\}$ on $D$ with $\int_{D} d \lambda_{j} \leq \int \eta d x$ and

$$
\int_{D}\left[w_{1}(x, T)-w_{2}(x, T)\right] \eta(x) d x=\int_{D} w_{1}\left(x, \tau_{j}\right)-w_{2}\left(x, \tau_{j}\right) d \lambda_{j}(x)
$$

where $w_{i}(x)=G u_{i}(x)=\int_{D} G(x, y) u_{i}(y) d y$ (i.e. the Green's potential of $u_{i}$ ), $i=1,2$.

Proof. The proof is contained in the proof of Lemma 2.12 of [DK1].

Let $u$ be a strong solution of the (IDP) and let $w=G u$. Then $\partial w / \partial t=$ $-u \leq 0$ (see (2.25) of [DK1]) and hence $\lim _{t \rightarrow 0} w(x, t)$ exists for each $x \in D$.

Lemma 2.1.2 (Pierre's maximum principle). Let $u_{1}, u_{2}$ be two strong solutions of the $(I D P)$ in $D \times(0, \infty)$. Suppose that $u_{2} \in C(\bar{D} \times[0, \infty))$ and that $w_{i}=G u_{i}$ verify $\lim _{t \rightarrow 0} w_{1}(x, t) \geq \lim _{t \rightarrow 0} w_{2}(x, t)$. Then $w_{1}(x, t) \geq w_{2}(x, t)$.

Proof. Same as the proof of Lemma 3 of [DK2].

Lemma 2.1.3. Let $u$ be a strong solution of the $(I D P)$ in $D \times(0, \infty)$ and $w=$ $G u$. Then there exists $x_{0} \in D$ such that $\lim _{t \rightarrow 0} w\left(x_{0}, t\right)<\infty$.

Proof. Suppose that $\lim _{t \rightarrow 0} w(x, t)=\infty \forall x \in D$. We claim that this implies that $w(x, t)=\infty \forall x \in D, t>0$. In order to prove the claim we first note that Lemma 2.1.2 implies that

$$
w(x, t) \geq w_{f}(x, t)=G u_{f} \quad \forall f \in C_{0}^{\infty}(D)
$$

where $u_{f}$ is the solution of $\Delta u_{f}=\partial_{t} u_{f}$ in $D \times(0, \infty)$ with initial data $u_{f}(x, 0)=f(x), \forall x \in D$ and boundary data $\left.u_{f}\right|_{\partial D \times(0, \infty)} \equiv 0$.

$$
\begin{aligned}
\Rightarrow w(x, t) & \geq w_{\lambda f}(x, t)=G u_{\lambda f}=G\left(\lambda u_{f}\right) \\
& =\lambda G u_{f} \quad \forall \lambda>0, f \in C_{0}^{\infty} .
\end{aligned}
$$

Now fix an $f \in C_{0}^{\infty}, f \geq 0$, and $f(\bar{x})>0$ for some $\bar{x} \in D$. Then

$$
u_{f}(x, t)>0 \quad \forall x \in D, t>0
$$

by the Harnack inequality. So $G u_{f}(x, t)>0$ and thus

$$
w(x, t) \geq \lambda G u_{f}(x, t) \rightarrow \infty \quad \text { as } \lambda \rightarrow \infty .
$$


On the other hand $\forall x \in D, t>0$,

$$
w(x, t)=\int_{D} G(x, y) u(y, t) d y \leq\|G(x, \cdot)\|_{L^{1}(D)}\|u(\cdot, t)\|_{L^{\infty}(D)}<\infty .
$$

Contradiction arises. Therefore there must exist an $x_{0} \in D$ such that

$$
\lim _{t \rightarrow 0} w\left(x_{0}, t\right)<\infty .
$$

Lemma 2.1.4. Let $u$ be a strong solution of the (IDP) in $D \times(0, \infty)$. Then

$$
\sup _{t>0} \int_{D} u(x, t) \delta(x) d x<\infty \text { and } \int_{D} h(x) d x<\infty
$$

where $h(x)=\lim _{t \rightarrow 0} w(x, t)$.

Proof. The proof follows the argument used in the proof of Lemma 6 in [DK2].

Theorem 2.1.5. Let $u$ be a strong solution of the (IDP). Then there is a positive Borel measure $\mu$ on $D$ with $\int_{D} \delta(x) d \mu(x)<\infty$ and a positive Borel measure $\lambda$ on $\partial D$ with $\int_{\partial D} d \lambda<\infty$ such that whenever $\eta \in C^{\infty}\left(R^{n}\right),\left.\eta\right|_{\partial D} \equiv 0$, we have

$$
\lim _{t \rightarrow 0} \int_{D} u(x, t) \eta(x) d x=\int_{D} \eta d \mu+\int_{\partial D} \frac{\partial \eta}{\partial N} d \lambda
$$

where $\partial / \partial N$ is the derivative in the direction of the inward normal $N$.

Proof. Let $h(x)=\lim _{t \rightarrow 0} w(x, t)$. By Lemma 6.1.4, $\int_{D} h(x) d x<\infty$. Hence $h$ is superharmonic in $D$ and we can use the same argument as in the proof of Theorem 7 in [DK2] to finish the proof of Theorem 2.1.5.

Theorem 2.1.6 (Uniqueness). Suppose $u_{1}$ and $u_{2}$ are two strong solutions of the (IDP) and that

$$
\begin{aligned}
\lim _{t \rightarrow 0} \int_{D} u_{1}(x, t) \eta(x) d x=\lim _{t \rightarrow 0} \int_{D} u_{2}(x, t) \eta(x) d x \\
\forall \eta \in C^{\infty}\left(R^{n}\right),\left.\eta\right|_{\partial D} \equiv 0 .
\end{aligned}
$$

Then $u_{1} \equiv u_{2}$.

Proof. The same as the proof of Theorem 8 of [DK2].

We need one more lemma whose proof is not hard.

Lemma 2.1.7. Let $G(x, t ; Q, s)$ be the Green kernel for the heat equation and $\eta \in C^{\infty}\left(R^{n}\right),\left.\eta\right|_{\partial D} \equiv 0$. Then

$$
\int_{x \in D} G(x, t ; Q, 0) \eta(x) d x \leq C \delta(Q)<\infty \quad \forall Q \in D, 0<t \leq 1,
$$

for some constant $C$ independent of $Q \in D$ and $0<t \leq 1$ where $\delta(Q)=$ $\operatorname{dist}(Q, \partial D)$.

Theorem 2.1.8 (Existence). Given a pair of measures $\mu$ on $D$ and $\lambda$ on $\partial D$ with $\mu \geq 0, \lambda \geq 0, \int_{D} \delta(x) d \mu(x)<\infty, \int_{\partial D} d \lambda<\infty$, there is a unique strong solution $u$ of the (IDP) such that for $\eta \in C^{\infty}\left(R^{n}\right),\left.\eta\right|_{\partial D} \equiv 0$, we have

$$
\lim _{t \rightarrow 0} \int_{D} u(x, t) \eta(x) d x=\int_{D} \eta d \mu+\int_{\partial D} \frac{\partial \eta}{\partial N} d \lambda
$$


where $\partial / \partial N$ is the derivative in the direction of the inward normal $N$. In fact $u(x, t)$ is given explicitly by

$$
u(x, t)=\int_{Q \in D} G(x, t ; Q, 0) d \mu(Q)+\int_{Q \in \partial D} \frac{\partial G}{\partial N_{Q}}(x, t ; Q, 0) d \lambda(Q)
$$

where $G(x, t ; Q, s)$ is the Green kernel for the heat equation.

Proof. Let

$$
\begin{aligned}
& u_{1}(x, t)=\int_{Q \in D} G(x, t ; Q, 0) d \mu(Q) \\
& u_{2}(x, t)=\int_{Q \in \partial D} \frac{\partial G}{\partial N_{Q}}(x, t ; Q, 0) d \lambda(Q) .
\end{aligned}
$$

By the uniqueness theorem, it suffices to show that both $u_{1}$ and $u_{2}$ are strong solutions of (IDP) and that $u_{1}+u_{2}$ has initial trace $\mu$ on $D$ and $\lambda$ on $\partial D$. Clearly both $u_{1}$ and $u_{2}$ are continuous, nonnegative functions on $D \times(0, \infty)$. Since

$$
\begin{aligned}
\left|\int_{Q \in D} G(x, t ; Q, 0) d \mu(Q)\right| & \leq C \int_{D} \frac{\delta(Q)}{t^{n+1 / 2}} e^{-c|x-Q|^{2} / t} d \mu(Q) \\
& \leq \frac{C}{t^{n+1 / 2}} \int_{D} \delta(Q) d \mu(Q)
\end{aligned}
$$

and

$$
\begin{aligned}
\int_{\partial D}\left|\frac{\partial G}{\partial N_{q}}(x, t ; Q, 0)\right| d \lambda(Q) & \leq \int_{\partial D} \frac{C}{t^{n+1 / 2}} e^{-c|x-Q|^{2} / t} d \lambda(Q) \\
& \leq \frac{C}{t^{n+1 / 2}} \int_{\partial D} d \lambda(Q)<\infty
\end{aligned}
$$

we have $\forall \psi \in C^{\infty}(\bar{D} \times(0, \infty)),\left.\psi\right|_{\partial D \times\left[\tau_{1}, \tau_{2}\right]} \equiv 0, \tau_{1}>0$,

$$
\begin{aligned}
& \iint_{D \times\left[\tau_{1}, \tau_{2}\right]}\left[u_{1} \Delta \psi+u_{1} \frac{\partial \psi}{\partial t}\right] d x d t \\
& =\int_{D} \iint_{D \times\left[\tau_{1}, \tau_{2}\right]} G(x, t ; Q, 0)\left[\Delta \psi+\frac{\partial \psi}{\partial t}\right] d x d t d \mu(Q) \\
& =\int_{D}\left(\int \int _ { D \times [ \tau _ { 1 } , \tau _ { 2 } ] } \left[\Delta_{x} G(x, t ; Q, 0) \psi(x, t)\right.\right. \\
& \left.\left.\quad-\frac{\partial G}{\partial t}(x, t ; Q, 0) \psi(x, t)\right] d x d t\right) d \mu(Q) \\
& \quad+\left.\int_{Q \in D} \int_{D} G(x, t ; Q, 0) \psi(x, t) d x\right|_{t=\tau_{1}} ^{t=\tau_{2}} d \mu(Q) \\
& =\int_{D} u_{1}\left(x, \tau_{2}\right) \psi\left(x, \tau_{2}\right) d x-\int_{D} u_{1}\left(x, \tau_{1}\right) \psi\left(x, \tau_{1}\right) d x
\end{aligned}
$$


and

$$
\begin{aligned}
& \iint_{D \times\left[\tau_{1}, \tau_{2}\right]}\left[u_{2} \Delta \psi+u_{2} \frac{\partial \psi}{\partial t}\right] d x d t \\
& =\int_{\partial D} \iint_{D \times\left[\tau_{1}, \tau_{2}\right]} \frac{\partial G}{\partial N_{Q}}(x, t ; Q, 0)\left[\Delta \psi+\frac{\partial \psi}{\partial t}\right] d x d t d \lambda(Q) \\
& =\int_{\partial D} \iint_{D \times\left[\tau_{1}, \tau_{2}\right]}\left[\frac{\partial}{\partial N_{Q}} \Delta_{x} G(x, t ; Q, 0) \psi(x, t)\right. \\
& \left.\quad-\frac{\partial}{\partial N_{Q}} \partial_{t} G(x, t ; Q, 0) \psi(x, t)\right] d x d t d \lambda(Q) \\
& \quad+\left.\int_{\partial D} \int_{D} \frac{\partial G}{\partial N_{Q}}(x, t ; Q, 0) \psi(x, t) d x\right|_{t=\tau_{1}} ^{t=\tau_{2}} d \lambda(Q) \\
& =\int_{D} u_{2}\left(x, \tau_{2}\right) \psi\left(x, \tau_{2}\right) d x-\int_{D} u_{2}\left(x, \tau_{1}\right) \psi\left(x, \tau_{1}\right) d x
\end{aligned}
$$

It remains to show that

(i) $\lim _{(x, t) \rightarrow\left(Q_{0}, t_{1}\right)} u_{1}(x, t)=\lim _{(x, t) \rightarrow\left(Q_{0}, t_{1}\right)} u_{2}(x, t)=0 \quad \forall Q_{0} \in \partial D, t_{1}>0$,

and (ii): $u_{1}$ has trace $\mu$ on $D, 0$ on $\partial D$, and $u_{2}$ has trace 0 on $D, \lambda$ on $\partial D$.

Proof of (i).

$$
\begin{aligned}
u_{1}(x, t) & =\int_{Q \in D} G(x, t ; Q, 0) d \mu(Q) \\
& =\int_{Q \in D} \frac{G(x, t ; Q, 0)}{\delta(Q)} \delta(Q) d \mu(Q) .
\end{aligned}
$$

By Lemma 1.3,

$$
G(x, t ; Q, 0) \leq C_{T} \frac{\delta(x) \delta(Q)}{t^{n+2 / 2}} e^{-c|x-Q|^{2} / t} \text { for } 0<t<T
$$

for any $T>0$. The Lebesgue dominated convergence theorem then implies that $u_{1}(x, t) \rightarrow 0$ as $(x, t) \rightarrow\left(Q_{0}, t_{1}\right), Q_{0} \in \partial D, t_{1}>0$. That is,

$$
\lim _{(x, t) \rightarrow\left(Q_{0}, t_{1}\right)} u_{1}(x, t)=0 \forall Q_{0} \in \partial D, t_{1}>0 .
$$

Similarly $u_{2}(x, t) \rightarrow 0$ as $(x, t) \rightarrow\left(Q_{0}, t_{1}\right), \forall Q_{0} \in \partial D, t_{1}>0$. Hence $u_{1}$ and $u_{2}$ are both strong solutions of the (IDP).

Proof of (ii). By Fubini's theorem for any $\eta \in C^{\infty}\left(R^{n}\right),\left.\eta\right|_{\partial D} \equiv 0$,

$$
\begin{aligned}
\int_{x \in D} u_{1}(x, t) \eta(x) d x & =\int_{x \in D} \int_{Q \in D} G(x, t ; Q, 0) d \mu(Q) \eta(x) d x \\
& =\int_{Q \in D}\left(\int_{x \in D} G(x, t ; Q, 0) \eta(x) d x\right) d \mu(Q) \\
& \rightarrow \int_{Q \in D} \eta d \mu \text { by Lemma } 1.7
\end{aligned}
$$


Also

$$
\begin{aligned}
\int_{D} u_{2}(x, t) \eta(x) d x & =\int_{Q \in \partial D} \int_{x \in D} \frac{\partial G}{\partial N_{Q}}(x, t ; Q, 0) \eta(x) d x d \lambda(Q) \\
& =\int_{Q \in \partial D} \frac{\partial}{\partial N_{Q}}\left(\int_{x \in D} G(x, t ; Q, 0) \eta(x) d x\right) d \lambda(Q) \\
& \rightarrow \int_{Q \in \partial D} \frac{\partial \eta}{\partial N_{Q}}(Q) d \lambda(Q) \text { as } t \rightarrow 0
\end{aligned}
$$

2.2. A priori estimate. For any point $S \in \partial D$, there exists a local $C^{\infty}$ space coordinate system $\phi_{S}: R^{n} \rightarrow R$ at $S$, i.e., there exists $r_{0}>0$ such that

$$
\begin{aligned}
D \cap B\left(S, r_{0}\right)= & \left\{Q=\left(Q^{\prime}, Q_{n}\right): Q^{\prime} \in R^{n-1}, Q_{n} \in R, Q_{n}>\phi_{S}\left(Q^{\prime}\right)\right\} \\
& \cap B\left(S, r_{0}\right), \\
\partial D \cap B\left(S, r_{0}\right)= & \left\{Q=\left(Q^{\prime}, Q_{n}\right): Q^{\prime} \in R^{n-1}, Q_{n} \in R, Q_{n}=\phi_{S}\left(Q^{\prime}\right)\right\} \\
& \cap B\left(S, r_{0}\right)
\end{aligned}
$$

and with the $\left\{Q_{n}=0\right\}$ plane being tangential to $\partial D$ at $S$ and the origin of the local space coordinate system is at $S$, i.e., $S=(0,0)$ in the local space coordinate at $S$.

Since $\partial D \in C^{\infty}, \partial D$ satisfies the interior cone condition at $S=(0,0)$, i.e., there exists $M>0, \alpha>0$, such that

$$
\Gamma(S)=\left\{Q=\left(Q^{\prime}, Q_{n}\right):\left|Q^{\prime}\right| \leq M Q_{n}, 0<Q_{n} \leq \alpha\right\} \subset D
$$

where $\left(Q^{\prime}, Q_{n}\right)$ is the local space coordinate of $Q$ with respect to the coordinate system $\left(S, \phi_{S}\right)$.

We define the nontangential approach to $(S, 0) \in \partial D \times\{0\}$ in $D \times(0, \infty)$ to be the space time cone

$$
\begin{aligned}
& \Gamma_{\beta}(S)=\left\{(Q, t)=\left(Q^{\prime}, Q_{n}, t\right) \in R^{n-1} \times R^{+} \times R^{+}:\right. \\
& \left.\left|Q^{\prime}\right| \leq M Q_{n}, Q_{n}=\beta t^{3 / 2} \leq \alpha\right\}
\end{aligned}
$$

where $\left(Q^{\prime}, Q_{n}\right)$ is the local space coordinate of $Q$ with respect to the coordinate system $\left(S, \phi_{S}\right)$.

Note that we can choose $M$ and $\alpha$ such that they are independent of $\phi_{S}$ and $S \in \partial D$ but depend only on $\partial D$ and $r_{0}$.

Lemma 2.2.1. $\forall \alpha>0, a>0$, there exists $C_{\alpha}=C(a, \alpha)>0$ such that

$$
e^{-a|x-z|^{2} / t} \leq C_{\alpha} e^{-a|x|^{2} /(2 t)} \quad \forall x \in R^{n}, t>0,|z| \leq \alpha \sqrt{t} .
$$

Lemma 2.2.2. Fix an $S \in \partial D$ and let $\phi=\phi_{S}: R^{n-1} \rightarrow R$ be the $C^{\infty}$ local coordinate system associated with $S$ as described at the beginning of this section, i.e. there exists $r_{0}>0$ such that

$$
\begin{aligned}
D \cap B\left(S, r_{0}\right)= & \left\{Q=\left(Q^{\prime}, Q_{n}\right): Q^{\prime} \in R^{n-1}, Q_{n} \in R, Q_{n}>\phi_{S}\left(Q^{\prime}\right)\right\} \\
& \cap B\left(S, r_{0}\right), \\
\partial D \cap B\left(S, r_{0}\right)= & \left\{Q=\left(Q^{\prime}, Q_{n}\right): Q^{\prime} \in R^{n-1}, Q_{n} \in R, Q_{n}=\phi_{S}\left(Q^{\prime}\right)\right\} \\
& \cap B\left(S, r_{0}\right) .
\end{aligned}
$$


Let $\nu$ be a positive Borel measure on $D$ with $\int_{D} \delta(x) d \nu(x)<\infty$ and $\operatorname{supp} \nu \subset$ $B\left(S, r_{0}\right)$. If $u(x, t)=\int_{D} G(x, t ; Q, 0) d \nu(Q)$, then

$$
u(x, t) \leq C M(\delta(Q) d \nu(Q))\left(Q_{0}\right) \quad \forall(x, t) \in \Gamma_{\beta}\left(Q_{0}\right), Q_{0} \in B\left(S, r_{0}\right) \cap \partial D,
$$

where $C$ is independent of $Q_{0} \in B\left(S, r_{0}\right) \cap \partial D$ and $\nu$.

$$
\begin{gathered}
M(f)\left(Q_{0}\right)=\sup _{\rho>0} \frac{1}{\left|B^{\prime}\left(Q_{0}^{\prime}, \rho\right)\right|} \iint_{\substack{\left|Q^{\prime}-Q_{0}^{\prime}\right| \leq \rho \\
Q=\left(Q^{\prime}, Q_{n}\right), Q^{\prime} \in R^{n-1}}} f(Q) d Q, \\
M(\delta(Q) d \nu(Q))\left(Q_{0}\right)=\sup _{\rho>0} \frac{1}{\left|B^{\prime}\left(Q_{0}^{\prime}, \rho\right)\right|} \iint_{\substack{\left|Q^{\prime}-Q_{0}^{\prime}\right| \leq \rho \\
Q=\left(Q^{\prime}, Q_{n}\right), Q^{\prime} \in R^{n-1}}}^{\delta(Q) d \nu(Q)}
\end{gathered}
$$

where $Q_{0}=\left(Q_{0}^{\prime}, \phi\left(Q_{0}^{\prime}\right)\right) \in \partial D \cap B\left(S, r_{0}\right), Q=\left(Q^{\prime}, Q_{n}\right)$ is the local space coordinate of $Q$ with respect to the local coordinate system $\left(S, \phi_{S}\right)$ at $S$ as described at the beginning of this section, and $B^{\prime}\left(Q_{0}^{\prime}, \rho\right)=\left\{Q^{\prime} \in R^{n-1}: \mid Q^{\prime}-\right.$ $\left.Q_{0}^{\prime} \mid \leq \rho\right\}$.

Proof. By Lemma 1.3,

$$
G(x, t ; Q, 0) \leq C \frac{\delta(x) \delta(Q)}{t^{n+2 / 2}} e^{-c|x-Q|^{2} / t} .
$$

So for any $(x, t) \in \Gamma_{\beta}\left(Q_{0}\right), Q_{0} \in B\left(S, r_{0}\right) \cap \partial D$,

$$
\begin{aligned}
u(x, t) & =\int_{Q \in D} G(x, t ; Q, 0) d \nu(Q) \\
& \leq C \frac{\delta(x)}{t^{3 / 2}} \cdot \frac{1}{t^{n-1 / 2}} \iint_{Q \in D} e^{-c|x-Q|^{2} / t} \delta(Q) d \nu(Q) \\
& \leq C^{\prime} \frac{1}{t^{n-1 / 2}} \iint_{Q \in D} e^{-c^{\prime}\left|Q_{0}-Q\right|^{2} / t} \delta(Q) d \nu(Q) \quad \text { by Lemma } 2.2 .1 \\
& \leq C^{\prime} \frac{1}{t^{n-1 / 2}}\left\{\iint_{\left|Q^{\prime}-Q_{0}^{\prime}\right| \leq t^{1 / 2}} e^{-c^{\prime}\left|Q_{0}^{\prime}-Q^{\prime}\right|^{2} / t} \delta(Q) d \nu(Q)\right. \\
& \left.\quad+\sum_{k=0}^{\infty} \iint_{2^{k} t^{1 / 2} \leq\left|Q^{\prime}-Q_{0}^{\prime}\right| \leq 2^{k+1} t^{1 / 2}} e^{-c^{\prime}\left|Q_{0}^{\prime}-Q^{\prime}\right|^{2} / t} \delta(Q) d \nu(Q)\right\} \\
& \leq C\left(1+\sum_{k=0}^{\infty}\left(2^{k+1}\right)^{n-1} e^{-c^{\prime} 2^{2 k}}\right) M(\delta(Q) d \nu(Q))\left(Q_{0}\right) \\
& \leq C^{\prime} M(\delta(Q) d \nu(Q))\left(Q_{0}\right) .
\end{aligned}
$$

Theorem 2.2.3. Let $u_{2}(x, t)=\int_{\partial D} \partial G / \partial N_{Q}(x, t ; Q, 0) d \lambda(Q)$ where $\lambda$ is a positive Borel measure on $\partial D$ with $\int_{\partial D} d \lambda<\infty$. Then $u_{2}(x, t) \leq C \widetilde{M}(d \lambda)\left(Q_{0}\right)$ for all $(x, t) \in \Gamma_{\beta}\left(Q_{0}\right), Q_{0} \in \partial D$, where

$$
\widetilde{M}(d \lambda)\left(Q_{0}\right)=\sup _{\rho>0} \frac{1}{\rho^{n-1}} \int_{\substack{\left|Q-Q_{0}\right| \leq \rho \\ Q \in \partial D}} d \lambda(Q), \quad Q_{0} \in \partial D
$$


Proof. By Lemma 1.3,

$$
\frac{\partial G}{\partial N_{Q}}(x, t ; Q, 0) \leq C \frac{\delta(x)}{t^{3 / 2}} \cdot \frac{1}{t^{n-1 / 2}} e^{-c|x-Q|^{2} / t} .
$$

So

$$
\begin{aligned}
u_{2}(x, t) & \leq C \frac{\delta(x)}{t^{3 / 2}} \cdot \frac{1}{t^{n-1 / 2}} \int_{Q \in \partial D} e^{-c|x-Q|^{2} / t} d \lambda(Q) \\
& \leq C \frac{1}{t^{n-1 / 2}} \int_{Q \in \partial D} e^{-c|x-Q|^{2} / t} d \lambda(Q) \quad \text { for }(x, t) \in \Gamma_{\beta}\left(Q_{0}\right) \\
& \leq C^{\prime} \widetilde{M}(d \lambda)\left(Q_{0}\right) \quad \forall(x, t) \in \Gamma_{\beta}\left(Q_{0}\right), \quad Q_{0} \in \partial D
\end{aligned}
$$

2.3. A Fatou theorem for the corner points of a cylinder. In this subsection we will prove the convergence a.e. on $\partial D \times\{0\}$ of the strong solution $u(x, t)$ of the heat equation in a cylinder as $(x, t) \rightarrow\left(Q_{0}, 0\right) \in \partial D \times\{0\}$ along the space time cone $\Gamma_{\beta}\left(Q_{0}\right)$ as defined in subsection 2.2 by assuming the everywhere convergence of the solution $\bar{u}$ of the heat equation at the corner points $\partial D \times\{0\}$ with initial trace $(d \mu, d \lambda)=(0, d \sigma)$ (where $d \sigma$ is the surface measure on $\partial D$ ) along these nontangential paths. We will use the same notation as in subsection 2.2 throughout this subsection.

Lemma 2.3.1. With the same notation as Lemma 2.2.2, there exists $C>0$ such that

$$
\left|\left\{Q_{0} \cap \partial D \cap B\left(S, r_{0}\right): \lambda \leq M(\delta(Q) d \nu(Q))\left(Q_{0}\right)\right\}\right| \leq \frac{C}{\lambda} \int_{B\left(S, r_{0}\right) \cap D} \delta(Q) d \nu(Q) .
$$

Proof. See [S, Chapter 1].

Theorem 2.3.2. Let $u_{1}=\int_{D} G(x, t ; Q, 0) d \mu(Q)$ with $\mu$ being a positive Borel measure on $D$ with $\int_{D} \delta(Q) d \mu(Q)<\infty$. Then

$$
\lim _{\substack{(x, t) \in \Gamma_{\beta}\left(Q_{0}\right) \\ t \rightarrow 0}} u_{1}(x, t)=0 \text { a.e. } Q_{0} \in \partial D .
$$

Proof. Note that in order to prove the theorem, it suffices to show that

$$
\lim _{\substack{(x, t) \in \Gamma_{\beta}\left(Q_{0}\right) \\ t \rightarrow 0}} u_{1}(x, t)=0 \quad \text { a.e. } Q_{0} \in \partial D \cap B\left(S, r_{0}\right)
$$

for any $S \in \partial D$ and $r_{0}$ is as described at the beginning of this subsection.

Hence without loss of generality, we fix an $S \in \partial D$ and assume from now on that $Q_{0} \in \partial D \cap B\left(S, r_{0}\right)$. Write

$$
E_{r}=B\left(S, r_{0}\right) \cap\{Q \in D: \delta(Q) \leq r\} \text {. }
$$

Then for all $(x, t) \in \Gamma_{\beta}\left(Q_{0}\right)$,

$$
\begin{aligned}
u_{1}(x, t)= & \int_{E_{r}^{c} \cap D} G(x, t ; Q, 0) d \mu(Q)+\int_{E_{r}} G(x, t ; Q, 0) d \mu(Q) \\
\leq & C \int_{Q \in E_{r}^{c} \cap D} \frac{\delta(x) \delta(Q)}{t^{n+2 / 2}} e^{-c|x-Q|^{2} / t} d \mu(Q) \\
& +C M\left(\delta(Q) \chi_{E_{r}} d \mu(Q)\right)\left(Q_{0}\right) \\
= & I_{1}+I_{2}
\end{aligned}
$$


by Lemma 1.3 and Lemma 2.2.2 where $\chi_{A}$ is the characteristic function of the set $A$.

By the Lebesgue dominated convergence theorem, $I_{1}$ vanishes as $(x, t) \quad(\in$ $\left.\Gamma_{\beta}\left(Q_{0}\right)\right) \rightarrow\left(Q_{0}, 0\right)$. Hence for any $\lambda>0$,

$$
\begin{aligned}
& \left|\left\{Q_{0} \in \partial D \cap B\left(S, r_{0}\right): \lambda \leq \varlimsup_{\substack{(x, t) \in \Gamma_{\beta}\left(Q_{0}\right) \\
t \rightarrow 0}} u_{1}(x, t)\right\}\right| \\
& \quad \leq\left|\left\{Q_{0} \in \partial D \cap B\left(S, r_{0}\right): \lambda \leq C M\left(\delta(Q) \chi_{E_{r}} d \mu(Q)\right)\left(Q_{0}\right)\right\}\right| \\
& \quad \leq \frac{C}{\lambda} \int_{Q \in B\left(S, r_{0}\right) \cap D} \delta(Q) \chi_{E_{r}} d \mu(Q) \\
& \quad \leq \frac{C}{\lambda} \int_{\substack{Q \in B\left(S, r_{0}\right) \cap D \\
\delta(Q) \leq r}} \delta(Q) d \mu(Q) \rightarrow 0 \quad \text { as } r \rightarrow 0
\end{aligned}
$$

since $\int_{B\left(S, r_{0}\right) \cap D} \delta(Q) d \mu(Q) \leq \int_{D} \delta(Q) d \mu(Q)<\infty$. Hence

$$
\left|\left\{Q_{0} \in \partial D \cap B\left(S, r_{0}\right): \lambda \leq \varlimsup_{\substack{(x, t) \in \Gamma_{\beta}\left(Q_{0}\right) \\ t \rightarrow 0}} u_{1}(x, t)\right\}\right|=0 \quad \forall \lambda>0 .
$$

Therefore

$$
\lim _{\substack{(x, t) \in \Gamma_{\beta}\left(Q_{0}\right) \\ t \rightarrow 0}} u_{1}(x, t)=0 \quad \text { a.e. } Q_{0} \in \partial D .
$$

Lemma 2.3.3. Let $\lambda$ be a positive Borel measure on $\partial D$ with $\int_{\partial D} d \lambda<\infty$ and let

$$
u_{2}(x, t)=\int_{\partial D} \frac{\partial G}{\partial N_{Q}}(x, t ; Q, 0) d \lambda(Q) .
$$

Then for almost every $Q_{0} \in \partial D$ we have

$$
\lim _{\substack{(x, t) \in \Gamma_{\beta}\left(Q_{0}\right) \\ t \rightarrow 0}}\left|u_{2}(x, t)-f\left(Q_{0}\right) \int_{\partial D} \frac{\partial G}{\partial N_{Q}}(x, t ; Q, 0) d \sigma(Q)\right|=0
$$

where $d \sigma$ is the surface measure on $\partial D$ and $f=d \lambda / d \sigma$ is the Radon-Nikodym derivative of $d \lambda$ with respect to $d \sigma$. (Note that $\int_{\partial D} f d \sigma \leq \int_{\partial D} d \lambda<\infty$ and $f \geq 0$ a.e. $[d \sigma]$.)

Proof. Write $d \lambda=f d \sigma+d \nu_{s}$ where $d \nu_{s} \perp d \sigma$. We will assume from now on that $(x, t) \in \Gamma_{\beta}\left(Q_{0}\right)$ in the proof. Now since $d \nu_{s} \perp d \sigma$,

$$
\lim _{r \rightarrow 0} \frac{1}{r^{n-1}} \int_{\substack{Q-Q_{0} \mid \leq r \\ Q \in \partial D}}\left|d \nu_{s}\right|(Q)=0 \text { a.e. } Q_{0} \in \partial D .
$$

Also a.e. $Q_{0} \in \partial D$ are Lebesgue points of $f$. Let $Q_{0} \in \partial D$ be a Lebesgue point of $f$ satisfying

$$
\lim _{r \rightarrow 0} \frac{1}{r^{n-1}} \int_{\substack{\left|Q-Q_{0}\right| \leq r \\ Q \in \partial D}}\left|d \nu_{s}\right|(Q)=0 \text { and } 0 \leq f\left(Q_{0}\right)<\infty .
$$


Then $\forall \varepsilon>0, \exists \delta>0$ such that

$$
\frac{1}{r^{n-1}} \int_{\substack{\left|Q-Q_{0}\right| \leq r \\ Q \in \partial D}}\left|d \nu_{s}\right|(Q) \leq \varepsilon
$$

and

$$
\frac{1}{r^{n-1}} \int_{\substack{\left|Q-Q_{0}\right| \leq r \\ Q \in \partial D}}\left|f(Q)-f\left(Q_{0}\right)\right| d \sigma(Q) \leq \varepsilon \quad \forall 0<r \leq \delta .
$$

Choose $k_{0} \in Z^{+}, k_{0}=k_{0}(t)$, such that $2^{k_{0}} t^{1 / 2} \leq \delta<2^{k_{0}+1} t^{1 / 2}$. Then

$$
\begin{aligned}
& \left|u_{2}(x, t)-f\left(Q_{0}\right) \int_{\partial D} \frac{\partial G}{\partial N_{Q}}(x, t ; Q, 0) d \sigma(Q)\right| \\
& \leq \int_{\substack{\left|Q-Q_{0}\right| \leq t^{1 / 2} \\
Q \in \partial D}} \frac{\partial G}{\partial N_{Q}}(x, t ; Q, 0)\left(\left|f(Q)-f\left(Q_{0}\right)\right| d \sigma(Q)+\left|d \nu_{s}\right|(Q)\right) \\
& \quad+\sum_{k=0}^{k_{0}} \int_{2^{k} t^{1 / 2} \leq\left|Q-Q_{0}\right| \leq 2^{k+1} t^{1 / 2}} \frac{\partial G}{\partial N_{Q}}(x, t ; Q, 0) \\
& \quad+\int_{\substack{\left|Q-Q_{0}\right| \geq \delta \\
Q \in \partial D}} \frac{\partial G}{\partial N_{Q}}(x, t ; Q, 0)\left(\left(f(Q)-f\left(Q_{0}\right)\right) d \sigma(Q)+d \nu_{s}(Q)\right)
\end{aligned}
$$

Since $|x-Q|=\left|x-Q_{0}+Q_{0}-Q\right|$ and $(x, t) \in \Gamma_{\beta}\left(Q_{0}\right) \Rightarrow \delta(x) \leq\left|x-Q_{0}\right| \leq$ $C t^{3 / 2} \leq C \sqrt{t}$, by Lemma 2.2.1 and Lemma 1.3 the last term above is dominated by

$$
\begin{aligned}
& C \frac{\delta(x)}{t^{n+2 / 2}} \int_{\substack{\left|Q-Q_{0}\right| \geq \delta \\
Q \in \partial D}} e^{-c|x-Q|^{2} / t}\left(d \lambda(Q)+\left|f\left(Q_{0}\right)\right| d \sigma(Q)\right) \\
& \quad \leq \frac{C^{\prime}}{t^{n-1 / 2}} \int_{\substack{\left|Q-Q_{0}\right| \geq \delta \\
Q \in \partial D}} e^{-c\left|Q_{0}-Q\right|^{2} / t}\left(d \lambda(Q)+\left|f\left(Q_{0}\right)\right| d \sigma(Q)\right) \\
& \quad \leq \frac{C}{t^{n-1 / 2}} e^{-c^{\prime} \delta^{2} / t}\left|\int_{\partial D} d \lambda(Q)+\right| f\left(Q_{0}\right)|d \sigma(Q)| \rightarrow 0 \text { as } t \rightarrow 0 .
\end{aligned}
$$


While the first two terms are dominated by

$$
\begin{aligned}
& C \frac{\delta(x)}{t^{3 / 2}} \cdot \frac{1}{t^{n-1 / 2}} \int_{\substack{Q-Q_{0} \mid \leq t^{1 / 2} \\
Q \in \partial D}} e^{-c|x-Q|^{2} / t}\left(\left|f(Q)-f\left(Q_{0}\right)\right| d \sigma(Q)+\left|d \nu_{s}\right|(Q)\right) \\
& \quad+C \frac{\delta(x)}{t^{3 / 2}} \cdot \sum_{k=0}^{k_{0}} \frac{\left(2^{k+1}\right)^{n-1}}{\left(2^{k+1} t^{1 / 2}\right)^{n-1}} \cdot \int_{2^{k} t^{1 / 2} \leq\left|Q-Q_{0}\right| \leq 2^{k+1} t^{1 / 2}} e^{-c|x-Q|^{2} / t} \\
& \quad \cdot\left(\left|f(Q)-f\left(Q_{0}\right)\right| d \sigma(Q)+\left|d \nu_{s}\right|(Q)\right) \\
& \leq \frac{C}{t^{n-1 / 2}} \int_{\left|Q-Q_{0}\right| \leq t^{1 / 2}}\left(\left|f(Q)-f\left(Q_{0}\right)\right| d \sigma(Q)+\left|d \nu_{s}\right|(Q)\right) \\
& \quad+C \sum_{k=0}^{k_{0}} \frac{\left(2^{k+1}\right)^{n-1}}{\left(2^{k+1} t^{1 / 2}\right)^{n-1}} \cdot \int_{\left|Q-Q_{0}\right| \leq 2^{k+1} t^{1 / 2}}^{Q \in \partial D} e^{-c|x-Q|^{2} / t} \\
& \leq C\left(1+\sum_{k=0}^{\infty} 2^{(k+1)(n-1)} e^{-c^{\prime} 2^{2 k}}\right) \cdot\left(\left|f(Q)-f\left(Q_{0}\right)\right| d \sigma(Q)+\left|d \nu_{s}\right|(Q)\right) \\
& \quad\left(C^{\prime} \varepsilon .\right.
\end{aligned}
$$

Therefore

$$
\varlimsup_{\substack{(x, t) \in \Gamma_{\beta}\left(Q_{0}\right) \\ t \rightarrow 0}}\left|u_{2}(x, t)-f\left(Q_{0}\right) \int_{\partial D} \frac{\partial G}{\partial N_{Q}}(x, t ; Q, 0) d \sigma(Q)\right| \leq C^{\prime} \varepsilon
$$

for all $\varepsilon>0$.

$$
\Rightarrow \lim _{(x, t) \in \Gamma_{\beta}\left(Q_{0}\right)}\left|u_{2}(x, t)-f\left(Q_{0}\right) \int_{\partial D} \frac{\partial G}{\partial N_{Q}}(x, t ; Q, 0) d \sigma(Q)\right|=0
$$

and the proof is completed.

Theorem 2.3.4. Let $\bar{u}(x, t)=\int_{\partial D} \partial G / \partial N_{Q}(x, t ; Q, 0) d \sigma(Q)$ with $d \sigma$ as the surface measure on $\partial D$ and $\Gamma_{\beta}\left(Q_{0}\right)$ as in subsection 2.2. Then

$$
\lim _{(x, t) \in \Gamma_{\beta}\left(Q_{0}\right)} \bar{u}(x, t)=\frac{\beta}{\sqrt{4 \pi}} \forall Q_{0} \in \partial D .
$$

Proof. Same as the proof of Theorem 2.4.1 of subsection 2.4.

Theorem 2.3.5. Let $u(x, t)$ be a strong solution of the $(I D P)$ in $D \times(0, \infty)$ with initial trace $\mu$ on $D$ and $\lambda$ on $\partial D$. (See Theorem 2.1.8 for its existence.) Then

$$
\lim _{\substack{(x, t) \in \Gamma_{\beta}\left(Q_{0}\right) \\ t \rightarrow 0}} u(x, t)=\frac{\beta}{\sqrt{4 \pi}} \cdot \frac{d \lambda}{d \sigma}\left(Q_{0}\right) \quad \text { a.e. } Q_{0} \in \partial D
$$

where $d \lambda / d \sigma$ is the Radon-Nikodym derivative of $d \lambda$ with respect to the surface measure $d \sigma$ on $\partial D$ and $\Gamma_{\beta}\left(Q_{0}\right)$ is as defined in subsection 2.2. 
Proof. By Theorems 2.1.5, 2.1.6, 2.1.8, $u(x, t)=u_{1}(x, t)+u_{2}(x, t)$ where

$$
\begin{aligned}
& u_{1}(x, t)=\int_{Q \in D} G(x, t ; Q, 0) d \mu(Q), \\
& u_{2}(x, t)=\int_{Q \in \partial D} \frac{\partial G}{\partial N_{Q}}(x, t ; Q, 0) d \lambda(Q) .
\end{aligned}
$$

By Theorem 2.3.2,

$$
\lim _{\substack{(x, t) \in \Gamma_{\beta}\left(Q_{0}\right) \\ t \rightarrow 0}} u_{1}(x, t)=0 \text { a.e. } Q_{0} \in \partial D .
$$

By Theorem 2.3.3 and Theorem 2.3.4,

$$
\lim _{\substack{(x, t) \in \Gamma_{\beta}\left(Q_{0}\right) \\ t \rightarrow 0}} u_{2}(x, t)=\frac{\beta}{\sqrt{4 \pi}} \cdot \frac{d \lambda}{d \sigma}\left(Q_{0}\right) \quad \text { a.e. } Q_{0} \in \partial D .
$$

Therefore

$$
\begin{aligned}
\lim _{\substack{(x, t) \in \Gamma_{\beta}\left(Q_{0}\right) \\
t \rightarrow 0}} u(x, t) & =\lim _{\substack{(x, t) \in \Gamma_{\beta}\left(Q_{0}\right) \\
t \rightarrow 0}} u_{1}(x, t)+\lim _{\substack{(x, t) \in \Gamma_{\beta}\left(Q_{0}\right) \\
t \rightarrow 0}} u_{2}(x, t) \\
& =\frac{\beta}{\sqrt{4 \pi}} \cdot \frac{d \lambda}{d \sigma}\left(Q_{0}\right)
\end{aligned}
$$

for a.e. $Q_{0} \in \partial D$.

2.4. A Fatou theorem for the solution with initial trace $(0, d \sigma)$. We will start this section with a definition. We let

$$
\begin{array}{r}
\bar{\Gamma}_{\beta}\left(Q_{0}\right)=\left\{(Q, s)=\left(Q^{\prime}, Q_{n}, s\right) \in R^{n-1} \times R^{+} \times R^{-}:\left|Q^{\prime}\right| \leq M Q_{n},\right. \\
\left.Q_{n}=\beta(-s)^{3 / 2} \leq \alpha\right\}
\end{array}
$$

where $Q_{0} \in \partial D$ and $Q=\left(Q^{\prime}, Q_{n}\right)$ is the local space coordinate of $Q \in D$ with respect to the coordinate system $\phi=\phi_{Q_{0}}$ at $Q_{0}$ as defined in subsection 2.2, $\alpha$ is the constant defined in the beginning of subsection 2.2 , and $M$ is the Lipschitz constant for $\partial D$. The main result of this section is the following theorem.

Theorem 2.4.1. Let $v(Q, s)=\int_{\partial D} \partial G / \partial N_{x}(x, 0 ; Q, s) d \sigma(x), s<0$, with $d \sigma$ being the surface measure on $\partial D$. Then

$$
\lim _{\substack{(Q, s) \in \bar{\Gamma}_{\beta}\left(Q_{0}\right) \\ s \rightarrow 0}} v(Q, s)=\frac{\beta}{\sqrt{4 \pi}} \forall Q_{0} \in \partial D .
$$

Before proving the theorem we would like to assume without loss of generality that $Q_{0}=0$ and $\phi$ is the local coordinate system at $Q_{0}=0$ with $\nabla \phi(0)=0$, $\phi \in C_{0}^{\infty}\left(R^{n-1}\right)$.

We first note that if $D^{\prime}$ is the image of $D$ under the transformation $\left(x^{\prime}, x_{n}\right)$ $\rightarrow\left(x^{\prime}, x_{n}^{\prime}\right)$ with $x_{n}^{\prime}=x_{n}-\phi\left(x^{\prime}\right), x=\left(x^{\prime}, x_{n}\right) \in D$ being the local coordinate of $x$ with respect to $(0, \phi)$, and if $\tilde{u}\left(\left(x^{\prime}, x_{n}^{\prime}\right), t\right)=u\left(\left(x^{\prime}, x_{n}^{\prime}+\phi\left(x^{\prime}\right)\right), t\right)$, $\left(x^{\prime}, x_{n}^{\prime}\right) \in D^{\prime}$, then

$$
\begin{gathered}
\Delta u-\partial_{t} u=0 \quad \text { in } D \times(-\infty, \infty) \\
\Leftrightarrow \operatorname{div}\left(a_{i j}\left(x^{\prime}\right) \nabla \tilde{u}\right)-\partial_{t} \tilde{u}=0 \quad \text { in } D^{\prime} \times(-\infty, \infty)
\end{gathered}
$$


where $a_{i j}\left(x^{\prime}\right)=\delta_{i j}, a_{1 j}\left(x^{\prime}\right)=a_{j 1}=-\partial \phi / \partial x_{j}, i, j=1, \ldots, n-1, a_{n n}=$ $1+\left|\nabla_{x^{\prime}} \phi\left(x^{\prime}\right)\right|^{2}$. It is not hard to see that $A\left(x^{\prime}\right)=\left(a_{i j}\right)$ is a uniformly elliptic matrix with the eigenvalues bounded above and below by positive constants independent of $x^{\prime}, \forall\left(x^{\prime}, x_{n}\right) \in D^{\prime}$, and depends on $\partial D$ only.

If $G(x, t ; y, \tau)=G\left(\left(x^{\prime}, x_{n}\right), t ;\left(y^{\prime}, y_{n}\right), \tau\right)$ is the Green's function for the heat equation $\Delta-\partial_{t}=0$ in $D \times(-\infty, \infty)$, then

$$
\widetilde{G}\left(\left(x^{\prime}, x_{n}^{\prime}\right), t ;\left(y^{\prime}, y_{n}^{\prime}\right), \tau\right)=G\left(\left(x^{\prime}, x_{n}^{\prime}+\phi\left(x^{\prime}\right)\right), t ;\left(y^{\prime}, y_{n}^{\prime}+\phi\left(y^{\prime}\right)\right), \tau\right)
$$

is the Green's function for $\operatorname{div}\left(a_{i j} \nabla\right)-\partial_{t}=0$ in $D^{\prime} \times(-\infty, \infty)$.

Idea of proof of Theorem 2.4.1. We first note that

$$
\int_{x \in \partial D} \frac{\partial G}{\partial N_{x}}(x, 0 ; y, \tau) d \sigma(x)=\left(\int_{\substack{x \in \partial D \\\left|x^{\prime}\right|>\varepsilon}}+\int_{\substack{x \in \partial D \\\left|x^{\prime}\right| \leq \varepsilon}}\right) \frac{\partial G}{\partial N_{x}}(x, 0 ; y, \tau) d \sigma(x) .
$$

The first term vanishes as $(y, \tau) \quad\left(\in \bar{\Gamma}_{\beta}(0)\right) \rightarrow 0$ by the Lebesgue dominated convergence theorem since its integrand

$$
\begin{aligned}
& \leq C \frac{\delta(y)}{|\tau|^{n+2 / 2}} \exp \left(-C \frac{|x-y|^{2}}{|\tau|}\right) \quad \text { by Lemma } 1.3 \\
& \leq \frac{C}{|\tau|^{n-1 / 2}} \exp \left(-C^{\prime} \frac{\varepsilon^{2}}{|\tau|}\right) \quad \text { for }(y, \tau) \in \bar{\Gamma}_{\beta}(0), \quad|y| \leq \frac{\varepsilon}{2} \\
& \rightarrow 0 \text { as } \tau \rightarrow 0 .
\end{aligned}
$$

While the second term

$$
\begin{aligned}
& =\int_{\substack{x \in \partial D \\
\left|x^{\prime}\right| \leq \varepsilon}} \frac{\partial G}{\partial N_{x}}(x, 0 ; y, \tau) d \sigma(x) \\
& =\int_{\substack{x \in \partial D \\
\left|x^{\prime}\right| \leq \varepsilon}} \nabla_{x} G(x, 0 ; y, \tau) \cdot\left(-\nabla_{x^{\prime}} \phi\left(x^{\prime}\right), 1\right) d x^{\prime} \\
& =-\int_{\substack{x \in \partial D \\
\left|x^{\prime}\right| \leq \varepsilon}} \nabla_{x^{\prime}} G(x, 0 ; y, \tau) \cdot \nabla_{x^{\prime}} \phi\left(x^{\prime}\right) d x^{\prime}+\int_{\substack{x \in \partial D \\
\left|x^{\prime}\right| \leq \varepsilon}} \frac{\partial G}{\partial x_{n}}(x, 0 ; y, \tau) d x^{\prime} .
\end{aligned}
$$

The term

$$
\begin{aligned}
& \left|\int_{\substack{x \in \partial D \\
\left|x^{\prime}\right| \leq \varepsilon}} \nabla_{x^{\prime}} G \cdot \nabla_{x^{\prime}} \phi d x^{\prime}\right| \\
& \quad \leq C \sup _{\left|x^{\prime}\right| \leq \varepsilon}\left\{\left|\nabla_{x^{\prime}}^{2} \phi\left(x^{\prime}\right)\right|\left|x^{\prime}\right|\right\} \cdot \int_{\substack{x \in \partial D \\
\left|x^{\prime}\right| \leq \varepsilon}} \frac{\delta(y)}{|\tau|^{n+2 / 2}} \exp \left(-C \frac{|x-y|^{2}}{|\tau|}\right) d x^{\prime} \\
& \quad \leq C \sup _{\left|x^{\prime}\right| \leq \varepsilon}\left|\nabla_{x^{\prime}}^{2} \phi\left(x^{\prime}\right)\right| \cdot \varepsilon \cdot \int_{\left|x^{\prime}\right| \leq \varepsilon} \frac{1}{|\tau|^{n-1 / 2}} \exp \left(-C^{\prime} \frac{\left|x^{\prime}\right|^{2}}{|\tau|}\right) d x^{\prime} \\
& \quad \leq C^{\prime} \varepsilon .
\end{aligned}
$$


Therefore it remains to consider

$$
\int_{\substack{x \in \partial D \\\left|x^{\prime}\right| \leq \varepsilon}} \frac{\partial G}{\partial x_{n}}(x, 0 ; y, \tau) d x^{\prime}=\int_{\substack{x \in \partial D \\\left|x^{\prime}\right| \leq \varepsilon}} \frac{\partial \widetilde{G}}{\partial x_{n}^{\prime}}(x, 0 ; y, \tau) d x^{\prime}
$$

and show that it converges to $\beta / \sqrt{4 \pi}$ as $(y, \tau) \quad\left(\in \bar{\Gamma}_{\beta}(0)\right) \rightarrow 0$. To show this we note that $\widetilde{G}(x, t ; y, s)=G_{y}(x, t ; y, s)+E(x, t ; y, s)$ where

$$
E(x, t ; y, s)=\int_{s}^{t} d \lambda \int_{D^{\prime}} G_{\eta}(x, t ; \eta, \lambda) \Phi(\eta, \lambda ; y, s) d \eta
$$

(See Lemma 2.4.6 below.) $G_{y}(x, t ; z, s)$ is the Green's function for

$$
L^{y} u=\sum a_{i j}\left(y^{\prime}\right) \frac{\partial^{2} u}{\partial x_{i} \partial x_{j}}-\frac{\partial u}{\partial t}=0, \quad y=\left(y^{\prime}, y_{n}^{\prime}\right),
$$

from which $\partial \widetilde{G} / \partial x_{n}=\partial G_{y} / \partial x_{n}+\partial E / \partial x_{n}$. Since $x \in \partial D^{\prime},\left|x^{\prime}\right| \leq \varepsilon \leq r_{0} \Rightarrow$ $x_{n}^{\prime}=0,\left.d \sigma\right|_{\partial D^{\prime} \cap\{|x| \leq \varepsilon\}}=d x^{\prime}$. If

$$
\int_{\substack{x \in \partial D \\\left|x^{\prime}\right| \leq \varepsilon}}\left|\nabla_{x} E\right| d \sigma(x) \rightarrow 0 \quad \text { as }(y, \tau)\left(\in \bar{\Gamma}_{\beta}(0)\right) \rightarrow 0
$$

then

$$
\lim _{(y, \tau) \in \bar{\Gamma}_{\beta}(0)} \int_{\substack{x \in \partial D^{\prime} \\\left|x^{\prime}\right| \leq \varepsilon}} \frac{\partial \widetilde{G}}{\partial x_{n}} d x^{\prime}=\lim _{\substack{(y, \tau) \in \bar{\Gamma}_{\beta}(0) \\ \tau \rightarrow 0}} \int_{\substack{x \in \partial D^{\prime} \\\left|x^{\prime}\right| \leq \varepsilon}} \frac{\partial G_{y}}{\partial x_{n}} d x^{\prime} .
$$

On the other hand, layer potential method implies that the function

$$
\Rightarrow w_{y}(x, 0 ; y, \tau)=\sum a_{i j}(y) n_{i}(x) \frac{\partial G_{y}}{\partial x_{j}}(x, 0 ; y, \tau)
$$

satisfies

$$
\begin{aligned}
w_{y}(x, 0 ; y, \tau)= & 2 \sum a_{i j}(y) n_{i}(x) \frac{\partial Z_{y}}{\partial x_{j}}(x, 0 ; y, \tau) \\
& -2 \int_{\tau}^{0} d \lambda \int_{\eta \in \partial D^{\prime}} H_{y}(x, 0 ; \eta, \lambda) w_{y}(\eta, \lambda ; y, \tau) d \sigma(\eta)
\end{aligned}
$$

where $Z_{y}(x, t ; \eta, \tau)$ is the fundamental solution for $L^{y} u=0$ and

$$
\begin{aligned}
H_{y}(x, t ; \eta, \lambda) & =\sum a_{i j}(y) n_{i}(x) \frac{\partial Z_{y}}{\partial x_{j}}(x, t ; \eta, \lambda) \\
\left(n_{i}(x)\right)_{i=1}^{n} & =N_{x}=\text { unit inward normal at } x \in \partial D^{\prime} \\
& =(0, \ldots, 0,1) \text { if }|x| \leq \varepsilon, x \in \partial D^{\prime}
\end{aligned}
$$

So

$$
\begin{aligned}
\sum a_{i j}(y) n_{i}(x) \frac{\partial G_{y}}{\partial x_{j}}(x, 0 ; y, \tau) & =\sum_{j} a_{n j}(y) \frac{\partial G_{y}}{\partial x_{j}}(x, 0 ; y, \tau) \\
& =\sum_{1 \leq j \leq n-1}-\frac{\partial \phi}{\partial y_{j}}\left(y^{\prime}\right) \cdot \frac{\partial G_{y}}{\partial x_{j}}+\frac{\partial G_{y}}{\partial x_{n}} .
\end{aligned}
$$


Therefore

$$
\begin{aligned}
& \left|\int_{\substack{x \in \partial D^{\prime} \\
\left|x^{\prime}\right| \leq \varepsilon}} \sum_{1 \leq j \leq n-1}-\frac{\partial \phi}{\partial y_{j}}\left(y^{\prime}\right) \frac{\partial G_{y}}{\partial x_{j}}(x, 0 ; y, \tau) d x^{\prime}\right| \\
& \quad \leq C \sup _{\left|x^{\prime}\right| \leq \varepsilon}\left|\nabla \phi\left(x^{\prime}\right)\right| \cdot \int_{\substack{x \in \partial D^{\prime} \\
\left|x^{\prime}\right| \leq \varepsilon}} \frac{\delta(y)}{|\tau|^{n+2 / 2}} \exp \left(-C \frac{|x-y|^{2}}{|\tau|}\right) d x^{\prime} \leq C^{\prime} \varepsilon
\end{aligned}
$$

as before. Hence

$$
\begin{aligned}
\int_{x \in \partial D} & \frac{\partial G}{\partial N_{x}} d \sigma(x) \approx \int_{\substack{x \in D^{\prime} \\
\left|x^{\prime}\right| \leq \varepsilon}} \frac{\partial G}{\partial x_{n}} d x=\int_{\substack{x \in \partial D^{\prime} \\
\left|x^{\prime}\right| \leq \varepsilon}} \frac{\partial \widetilde{G}}{\partial x_{n}^{\prime}} d x^{\prime} \\
\approx & \int_{\left|x^{\prime}\right| \leq \varepsilon} \frac{\partial G_{y}}{\partial x_{n}^{\prime}} d x^{\prime}+\int_{\left|x^{\prime}\right| \leq \varepsilon} \frac{\partial E}{\partial x_{n}^{\prime}} d x^{\prime} \\
\approx & \int_{\left|x^{\prime}\right| \leq \varepsilon} \frac{\partial G_{y}}{\partial x_{n}^{\prime}} d x^{\prime} \approx \int_{\left|x^{\prime}\right| \leq i} \sum a_{i j}(y) n_{i}(x) \frac{\partial G_{y}}{\partial x_{j}}(x, 0 ; y, \tau) d x^{\prime} \\
= & 2 \int_{\left|x^{\prime}\right| \leq \varepsilon} \sum a_{i j}(y) n_{i}(x) \frac{\partial Z_{y}}{\partial x_{j}}(x, 0 ; y, \tau) d x^{\prime} \\
& -2 \int_{\left|x^{\prime}\right| \leq \varepsilon} \int_{\tau}^{0} d \lambda \int_{\eta \in \partial D^{\prime}} H_{y}(x, 0 ; \eta, \lambda) w_{y}(\eta, \lambda ; y, \tau) d \sigma(\eta) d x^{\prime} \\
\rightarrow & \frac{\beta}{\sqrt{4 \pi}}
\end{aligned}
$$

if the first term above $\rightarrow \beta / \sqrt{4 \pi}$ and the second term above $\rightarrow 0$ as $(y, \tau)$ $\left(\in \bar{\Gamma}_{\beta}(0)\right) \rightarrow 0$.

By the above discussion, we see that in order to prove Theorem 2.4.1, it is natural to first investigate properties of $\widetilde{G}$. We would let $G_{y}(x, t ; \eta, \tau)$ be the Green's function for the equation

$$
L^{y} u=\sum a_{i j}(y) \frac{\partial^{2} u}{\partial x_{i} \partial x_{j}}(x, t)-\frac{\partial u}{\partial t}(x, t)=0 \quad \text { in } \partial D^{\prime} \times(-\infty, \infty)
$$

and

$$
\begin{aligned}
& Z_{y}(x, t ; \eta, \lambda) \\
& \quad=\frac{1}{\left(\operatorname{det} a^{i j}(y)\right)^{1 / 2}} \cdot \frac{1}{(4 \pi(t-\tau))^{n / 2}} \exp \left\{-\frac{\sum a^{i j}(y)\left(x_{i}-\eta_{i}\right)\left(x_{j}-\eta_{j}\right)}{4(t-\tau)}\right\}
\end{aligned}
$$

be its fundamental solution throughout this section where $\left(a_{i j}(x)\right)$ is as defined on page 624 and $\left(a^{i j}\right)=\left(a_{i j}\right)^{-1}$. We will also assume without loss of generality that $\partial D^{\prime}$ is smooth throughout this section. Since the adjoint equation for $L^{y}$ is

$$
L^{y^{*}} v=\sum a_{i j}(y) \frac{\partial^{2} v}{\partial z_{i} \partial z_{j}}(z, \tau)+\frac{\partial v}{\partial t}(z, \tau)=0,
$$

maximum principle holds for solutions of $L^{y}$ and $L^{y^{*}}$. Therefore Lemma 1.3 and Lemma 1.4 remains valid for $G_{y}$. For the sake of completeness, we state them here again. 
Lemma 2.4.2. For $-T \leq \tau<t \leq T, T>0$, we have

(i)

(ii)

$$
G_{y}(x, t ; z, \tau) \leq \frac{C}{(t-\tau)^{n / 2}} \exp \left(-C \frac{|x-z|^{2}}{t-\tau}\right), \quad x, z \in D^{\prime}
$$

$$
G_{y}(x, t ; z, \tau) \leq C \frac{\delta(z)}{(t-\tau)^{n+1 / 2}} \exp \left(-C \frac{|x-z|^{2}}{t-\tau}\right), \quad x, z \in D^{\prime}
$$

(iii)

(iv)

$$
G_{y}(x, t ; z, \tau) \leq C \frac{\delta(x) \delta(z)}{(t-\tau)^{n+2 / 2}} \exp \left(-C \frac{|x-z|^{2}}{t-\tau}\right), \quad x, z \in D^{\prime}
$$

$$
\begin{aligned}
& \left|\frac{\partial G_{y}}{\partial N_{x}}(x, t ; z, \tau)\right| \leq C \frac{\delta(z)}{(t-\tau)^{n+2 / 2}} \exp \left(-C \frac{|x-z|^{2}}{t-\tau}\right), \\
& x \in \partial D^{\prime}, \quad z \in D^{\prime} \text {, }
\end{aligned}
$$

where $\partial / \partial N_{x}$ is the derivative in the direction of the normal to $\partial D^{\prime}$ at the point $x \in \partial D^{\prime}, \delta(x)=\operatorname{dist}\left(x, \partial D^{\prime}\right)$, and $C$ is a constant independent of $x, z, t, \tau$.

Corollary 2.4.3. The following is true:

$$
\begin{aligned}
\left|\nabla_{z} G_{y}(x, t ; z, \tau)\right| & \leq C \frac{\delta(x)}{(t-\tau)^{n+2 / 2}} \exp \left(-C \frac{|x-z|^{2}}{t-\tau}\right), \\
\left|\nabla_{x} G_{y}(x, t ; z, \tau)\right| & \leq C \frac{\delta(z)}{(t-\tau)^{n+2 / 2}} \exp \left(-C \frac{|x-z|^{2}}{t-\tau}\right)
\end{aligned}
$$

for all $-T \leq \tau<t \leq T$ (for any fixed $T>0$ ), $x, z \in \overline{D^{\prime}}$, and $C$ is a constant independent of $y$ and depending only on $T>0$.

The following lemma comes from [LSU, Theorem 16.3].

Lemma 2.4.4. The Green's function $G_{y}(x, t ; z, \tau)$ for

$$
L^{y}=\sum a_{i j}(y) \partial_{x_{i} x_{j}}^{2}-\partial_{t}=0 \text { in } D^{\prime} \times(-\infty, \infty)
$$

satisfies the following inequalities:

$$
\begin{aligned}
& \left|\partial_{t}^{r} \partial_{x}^{s} G_{y}(x, t ; z, \tau)\right| \leq \frac{C}{(t-\tau)^{n+2 r+s / 2}} \exp \left(-C \frac{|x-z|^{2}}{t-\tau}\right), \\
& \left|\partial_{t}^{r} \partial_{x}^{s} G_{y}(x, t ; z, \tau)-\partial_{t^{\prime}}^{r} \partial_{x}^{s} G_{y}\left(x, t^{\prime} ; z, \tau\right)\right| \\
& \quad \leq C\left(t-t^{\prime}\right)^{3 / 2-r-s / 2}\left(t^{\prime}-\tau\right)^{-(n+3) / 2} \exp \left(-C \frac{|x-z|^{2}}{t-\tau}\right),
\end{aligned}
$$

where $2 r+s=1,2$ and $\tau<t^{\prime}<t$, and

$$
\begin{aligned}
& \left|\partial_{t}^{r} \partial_{x}^{s} G_{y}(x, t ; z, \tau)-\partial_{t}^{r} \partial_{\bar{x}}^{s} G_{y}(\bar{x}, t ; z, \tau)\right| \\
& \quad \leq C \frac{|x-\bar{x}|}{(t-\tau)^{n+3 / 2}} \exp \left(-C \frac{\left|x^{\prime \prime}-z\right|^{2}}{t-\tau}\right)
\end{aligned}
$$

where $2 r+s=2$ and $\bar{x}$ is that one of the points $x, x^{\prime}$ which is closest to $y$, $C$ is a positive constant independent of $y$.

The following lemma comes from [F, Chapter 1, Lemma 3]. 
Lemma 2.4.5. If $-\infty<\alpha<n / 2+1,-\infty<\beta<n / 2+1$, then

$$
\begin{aligned}
& \int_{\sigma}^{t} \int_{R^{n}}(t-\lambda)^{-\alpha} \exp \left(-\frac{h|x-\eta|^{2}}{4(t-\lambda)}\right)(\lambda-\sigma)^{-\beta} \exp \left(-\frac{h|\eta-y|^{2}}{4(\lambda-\sigma)}\right) d \eta d \lambda \\
& \quad=\left(\frac{4 \pi}{h}\right)^{n / 2} B\left(\frac{n}{2}-\alpha+1, \frac{n}{2}-\beta+1\right)(t-\sigma)^{n / 2+1-\alpha-\beta} \exp \left(-\frac{h|x-y|^{2}}{4(t-\sigma)}\right)
\end{aligned}
$$

where $B(a, b)=\Gamma(a) \Gamma(b) / \Gamma(a+b), \Gamma$ is the gamma function.

Lemma 2.4.6. If $\widetilde{G}(x, t ; y, \tau)$ is the Green's function for $L u=\operatorname{div}\left(a_{i j}\left(x^{\prime}\right) \nabla u\right)-$ $\partial_{t} u=0$ in $D^{\prime} \times(-\infty, \infty),\left(x^{\prime}, x_{n}^{\prime}\right) \in D^{\prime}$. Then the following integral identity holds:

$$
\begin{aligned}
& \widetilde{G}(x, t ; y, \tau)=G_{y}(x, t ; y, \tau)+E(x, t ; y, \tau), \\
& E(x, t ; y, \tau)=\int_{\tau}^{t} d \lambda \int_{D^{\prime}} G_{\eta}(x, t ; \eta, \lambda) \Phi(\eta, \lambda ; y, \tau) d \eta
\end{aligned}
$$

with $\Phi$ being determined from the integral identity

$$
\begin{aligned}
\Phi(x, t ; y, \tau)= & L G_{y}(x, t ; y, \tau) \\
& +\int_{\tau}^{t} d \lambda \int_{D^{\prime}} L G_{\eta}(x, t ; \eta, \lambda) \Phi(\eta, \lambda ; y, \tau) d \eta .
\end{aligned}
$$

Proof. The proof is exactly the same as the construction of the fundamental solution of $L u=0$ given in [F, Chapter 1] except that we use Lemma 2.4.4 to control $G_{y}$ and replace Theorem 1 of Chapter 1 of $[\mathrm{F}]$ by the following lemma. We omit the details of its proof.

Lemma 2.4.7. Let $f(y, \tau)$ be a continuous bounded function in $D^{\prime} \times\left(T_{0}, T_{1}\right)$. Then $J(x, t, \tau)=\int_{D^{\prime}} G_{y}(x, t ; y, \tau) f(y, \tau) d y$ is a continuous function in $(x, t, \tau), x \in \overline{D^{\prime}}, T_{0} \leq \tau<t \leq T_{1}$ and $\lim _{\tau \rightarrow t} J(x, t, \tau)=f(x, t)$.

Corollary 2.4.8. Let $v_{R}$ be the solution of the adjoint equation

$$
L^{y^{*}} v_{R}=\sum a_{i j}\left(y^{\prime}\right) \frac{\partial^{2} v_{R}}{\partial z_{i} \partial z_{j}}(z, \tau)+\frac{\partial v_{R}}{\partial \tau}(z, \tau)=0 \quad \text { in } D_{R} \times\left(-2 R^{2}, 0\right)
$$

of $L^{y}$ where $D_{R}=B(0,2 R) \backslash \overline{B(0, R)}$ with boundary value $\phi\left(z / R, \tau / R^{2}\right), \phi \in$ $C^{\infty}, 0 \leq \phi \leq 1$, and

$$
\begin{cases}\phi(z, \tau) \equiv 1 & \text { for }(z, \tau) \in \partial B(0,2) \times(-4,0] \cup(B(0,2) \backslash \overline{B(0,1)}) \times\{0\}, \\ \phi(z, \tau) \equiv 0 & \text { for }(z, \tau) \in \partial B(0,1) \times(-2,-4) .\end{cases}
$$

Then

$$
\left\|\nabla_{z} v_{R}\right\|_{L^{\infty}\left(D_{R} \times\left(-4 R^{2}, 0\right)\right)} \leq C / R<\infty
$$

for some constant $C$ independent of $y \in D^{\prime}$ and $R>0$.

Proof. The lemma follows from the boundary Schauder's estimate. (See [F, Theorem 6, p. 65].)

Lemma 2.4.9. With the same notation as in Lemma 2.4.6, then the function

$$
\begin{aligned}
F(x, t ; z, \tau) & =L G_{y}(x, t ; z, \tau) \\
& =\operatorname{div}\left(a_{i j}\left(x^{\prime}\right) \nabla_{x} G_{y}(x, t ; z, \tau)\right)-\partial_{t} G_{y}(x, t ; z, \tau)
\end{aligned}
$$


satisfies the adjoint equation $L^{y^{*}} F=0$ where

and

$$
\begin{gathered}
L^{y^{*}}=\sum a_{i j}\left(y^{\prime}\right) \frac{\partial^{2}}{\partial z_{i} \partial z_{j}}+\frac{\partial}{\partial \tau} . \\
\left.F(x, t ; z, \tau)\right|_{\substack{z \in \partial D^{\prime} \\
t>\tau}} \equiv 0
\end{gathered}
$$

(i) $|F(x, t ; z, \tau)| \leq C\left\{\frac{|x-y|}{(t-\tau)^{n+2 / 2}}+\frac{1}{(t-\tau)^{n+1 / 2}}\right\} \cdot \exp \left\{-C \frac{|x-z|^{2}}{t-\tau}\right\}$,

$$
|F(x, t ; z, \tau)|
$$

(ii)

$$
\leq C_{\alpha}\left\{\frac{|x-y| \delta(z)^{1-\alpha}}{(t-\tau)^{(n+3-\alpha) / 2}}+\frac{\delta(z)^{1-\alpha}}{(t-\tau)^{(n+2-\alpha) / 2}}\right\} \cdot \exp \left\{-C \frac{|x-z|^{2}}{t-\tau}\right\}
$$

for some constants $C_{\alpha}, C>0, C_{\alpha}, C$ both independent of $y \in \partial D^{\prime},-1<\tau<$ $t<1, x, z \in \partial D^{\prime}, 0 \leq \alpha<1$.

Proof. We first note that

$$
L^{y^{*}} F(x, t ; z, \tau)=L^{y^{*}} L G_{y}(x, t ; z, \tau)=0
$$

and

$$
\begin{aligned}
& \left.G_{y}(x, t ; z, \tau)\right|_{\substack{z \in \partial D^{\prime} \\
t>\tau}}=0 \\
& \left.\quad \Rightarrow \partial_{x}^{r} \partial_{t}^{s} G_{y}(x, t ; z, \tau)\right|_{\substack{z \in \partial D^{\prime} \\
t>\tau}}=0 \quad \forall r, s=0,1,2, \ldots \\
& \quad \Rightarrow F(x, t ; z, \tau)=\left.L G_{y}(x, t ; z, \tau)\right|_{\substack{z \in \partial D^{\prime} \\
t>\tau}}=0 .
\end{aligned}
$$

Also

$$
\begin{aligned}
& |F(x, t ; z, \tau)| \\
& \quad=\left|L G_{y}(x, t ; z, \tau)\right| \\
& =\mid \sum a_{i j}\left(x^{\prime}\right) \frac{\partial^{2} G_{y}}{\partial x_{i} \partial x_{j}}(x, t ; z, \tau)-\Delta_{y^{\prime}} \phi\left(y^{\prime}\right) \frac{\partial G_{y}}{\partial x_{n}}(x, t ; z, \tau) \\
& \quad=\left|\sum\left(a_{i j}\left(x^{\prime}\right)-a_{i j}\left(y^{\prime}\right)\right) \frac{\partial^{2} G_{y}}{\partial x_{i} \partial x_{j}}(x, t ; z, \tau)-\Delta_{y^{\prime}} \phi\left(y^{\prime}\right) \frac{\partial G_{y}}{\partial x_{n}}(x, t ; z, \tau)\right| \\
& \leq C \frac{|x-y|}{(t-\tau)^{n+2 / 2}} \exp \left(-C \frac{|x-z|^{2}}{t-\tau}\right) \\
& \quad+C \frac{1}{(t-\tau)^{n+1 / 2}} \cdot \exp \left(-C \frac{|x-z|^{2}}{t-\tau}\right) .
\end{aligned}
$$

Proof of (ii). The proof of (ii) of Lemma 2.4.9 will be similar to the proof of (ii) of Lemma 1.4. We first note that since $\partial D^{\prime}$ is smooth, $\partial D^{\prime}$ satisfies the exterior sphere condition, i.e. there exists a positive constant $h>0$ such that for each $x \in \partial D^{\prime}, \forall 0<r \leq h$, there exists $x_{0} \in D^{\prime c}$ such that $\overline{B\left(x_{0}, r\right)} \cap \overline{D^{\prime}}=\{x\}$.

Case 1. $\delta(z) \geq h$, then

$$
1 \leq \frac{\delta(z)^{1-\alpha}}{h^{1-\alpha}} \leq \frac{\delta(z)^{1-\alpha}}{h^{1-\alpha}} \cdot \frac{(t-\tau)^{1-\alpha / 2}}{(t-\tau)^{1-\alpha / 2}} \leq C_{\alpha} \frac{\delta(z)^{1-\alpha}}{(t-\tau)^{1-\alpha / 2}}
$$


since $|t|,|\tau| \leq 1$. So by (i),

$$
\begin{aligned}
\mid F(x & , t ; z, \tau) \mid \\
& \leq C_{\alpha}\left\{\frac{|x-y|}{(t-\tau)^{n+2 / 2}}+\frac{1}{(t-\tau)^{n+1 / 2}}\right\} \exp \left(-C \frac{|x-z|^{2}}{t-\tau}\right) \frac{\delta(z)^{1-\alpha}}{(t-\tau)^{1-\alpha / 2}} \\
& \leq C_{\alpha}^{\prime}\left\{\frac{|x-y| \delta(z)^{1-\alpha}}{(t-\tau)^{n+3-\alpha / 2}}+\frac{\delta(z)^{1-\alpha}}{(t-\tau)^{n+2-\alpha / 2}}\right\} \exp \left(-C \frac{|x-z|^{2}}{t-\tau}\right) .
\end{aligned}
$$

Case 2. $\delta(z) \geq(t-\tau)^{1 / 2} / 4$. The proof is similar to the proof of Case 1 .

Case 3. $\delta(z) \leq h$ and $\delta(z) \leq(t-\tau)^{1 / 2} / 4 \leq h$.

We fix $z^{*} \in \partial D^{\prime}$ with $\left|z-z^{*}\right|=\delta(z)$ and set $R=(t-\tau)^{1 / 2} / 4$. Since $\partial D^{\prime}$ satisfies the exterior sphere condition, there exists $z_{0} \in \partial D^{\prime c}$ such that $\overline{B\left(z_{0}, R\right)} \cap \overline{D^{\prime}}=\left\{z^{*}\right\}$. We may also assume $\overline{z z^{*}} \subset D^{\prime}$. Consider the cylinder $\underline{Q}_{R}=D_{R} \times\left(\tau, \tau+(2 R)^{2}\right), D_{R}=B\left(z_{0}, 2 R\right) \backslash \overline{B\left(z_{0}, R\right)}$. Then, we have $(z, \tau) \in$ $\bar{Q}_{R} \cap \overline{D^{\prime}} \times(-1,1)$. Similar to the proof of Case 3 of Lemma 1.4, for any $(\bar{z}, \bar{\tau}) \in \bar{Q}_{R} \cap \overline{D^{\prime}} \times[-1,1]$

$$
|F(x, t ; \bar{z}, \bar{\tau})| \leq C\left\{\frac{|x-y|}{(t-\tau)^{n+2 / 2}}+\frac{1}{(t-\tau)^{n+1 / 2}}\right\} \exp \left(-C^{\prime} \frac{|x-z|^{2}}{t-\tau}\right) .
$$

If $\bar{v}_{R}(\bar{z}, \bar{\tau})=v_{R}\left(\bar{z}-z_{0}, \bar{\tau}-\left(\tau+4 R^{2}\right)\right),(\bar{z}, \bar{\tau}) \in \bar{Q}_{R}$, where $v_{R}$ is as in Corollary 2.4 .8 , then since

$$
\left\{\begin{array}{l}
F(x, t ; \bar{z}, \bar{\tau})=0 \\
\bar{v}_{R}(\bar{z}, \bar{\tau}) \geq 0 \quad \forall(\bar{z}, \bar{\tau}) \in \bar{Q}_{R} \cap \partial D^{\prime} \times(-\infty, \infty)
\end{array}\right.
$$

and

$$
\left\{\begin{array}{l}
|F(x, t ; \bar{z}, \bar{\tau})| \leq C\left\{\frac{|x-y|}{(t-\tau)^{n+2 / 2}}+\frac{1}{(t-\tau)^{n+1 / 2}}\right\} \exp \left(-C \frac{|x-z|^{2}}{t-\tau}\right), \\
\bar{v}_{R}(\bar{z}, \bar{\tau}) \equiv 1
\end{array}\right.
$$

for all

$(\bar{z}, \bar{\tau}) \in\left\{\partial B\left(z_{0}, 2 R\right) \times\left(\tau, \tau+(2 R)^{2}\right]\right.$

$$
\left.\cup\left(B\left(z_{0}, 2 R\right) \backslash B\left(z_{0}, R\right)\right) \times\left\{\tau+(2 R)^{2}\right\}\right\} \cap \bar{D} \times(-\infty, \infty),
$$

by the maximum principle applied to the functions $F(x, t, \cdot, \cdot)$ and

$$
C\left\{\frac{|x-y|}{(t-\tau)^{n+2 / 2}}+\frac{1}{(t-\tau)^{n+1 / 2}}\right\} \exp \left(-C \frac{|x-z|^{2}}{t-\tau}\right) \bar{v}_{R}(\cdot, \cdot)
$$

in the region $Q_{R} \cap D \times(-\infty, \infty)$, we have

$$
\begin{aligned}
|F(x, t ; z, \tau)| & \leq C\left\{\frac{|x-y|}{(t-\tau)^{n+2 / 2}}+\frac{1}{(t-\tau)^{n+1 / 2}}\right\} \exp \left(-C \frac{|x-z|^{2}}{t-\tau}\right) \bar{v}_{R}(z, \tau) \\
& \leq C \frac{\delta(z)}{(t-\tau)^{1 / 2}}\left\{\frac{|x-y|}{(t-\tau)^{n+2 / 2}}+\frac{1}{(t-\tau)^{n+1 / 2}}\right\} \exp \left(-C \frac{|x-z|^{2}}{t-\tau}\right) \\
& =C_{\alpha}\left\{\frac{|x-y| \delta(z)^{1-\alpha}}{(t-\tau)^{n+3-\alpha / 2}}+\frac{\delta(z)^{1-\alpha}}{(t-\tau)^{n+2-\alpha / 2}}\right\} \exp \left(-C \frac{|x-z|^{2}}{t-\tau}\right)
\end{aligned}
$$

(since $\left.\delta(z) \leq(t-\tau)^{1 / 2} / 4 \Rightarrow\left(\delta(z) /(t-\tau)^{1 / 2}\right)^{\alpha} \leq 4^{-\alpha}\right)$. 
Case 4. $\delta(z) \leq h$ and $\delta(z) \leq h \leq 4 h \leq(t-\tau)^{1 / 2}$.

Proof of Case 4 is similar to Case 3 . We omit the details.

Lemma 2.4.10. With the same notation as 2.4.6, the function $\Phi(x, t ; y, \tau)$ satisfies the inequality

$$
|\Phi(x, t ; y, \tau)| \leq C_{\alpha} \frac{\delta(y)^{1-\alpha}}{(t-\tau)^{n+2-\alpha / 2}} \exp \left(-C \frac{|x-y|^{2}}{t-\tau}\right), \quad 0<\alpha<1,
$$

where $C_{\alpha}$ is a constant independent of $x, y \in D^{\prime}$ and $t, \tau,-1<\tau<t<1$.

Proof. Since $\Phi$ satisfies the integral identity,

$$
\begin{aligned}
\Phi(x, t ; y, \tau)= & L G_{y}(x, t ; y, \tau) \\
& +\int_{\tau}^{t} d \lambda \int_{\partial D^{\prime}} L G_{\eta}(x, t ; \eta, \lambda) \Phi(\eta, \lambda ; y, \tau) d \eta .
\end{aligned}
$$

Therefore $\Phi(x, t ; y, \tau)=\sum_{\nu=1}^{\infty}\left(L G_{y}\right)_{\nu}(x, t ; y, \tau)$ where $\left(L G_{y}\right)_{1}(x, t ; y, \tau)$ $=L G_{y}(x, t ; y, \tau)$ and

$$
\left(L G_{y}\right)_{\nu+1}(x, t ; y, \tau)=\int_{\tau}^{t} d \lambda \int_{\partial D^{\prime}} L G_{\eta}(x, t ; \eta, \lambda)\left(L G_{y}\right)_{\nu}(\eta, \lambda ; y, \tau) d \eta
$$

for all $\nu=1,2, \ldots$. By Lemma 2.4.9,

$$
\begin{aligned}
& \left|L G_{y}(x, t ; y, \tau)\right|(=|F(x, t ; y, \tau)|) \\
& \quad \leq C_{\alpha}\left\{\frac{|x-y| \delta(y)^{1-\alpha}}{(t-\tau)^{n+3-\alpha / 2}}+\frac{\delta(y)^{1-\alpha}}{(t-\tau)^{n+2-\alpha / 2}}\right\} \exp \left(-C \frac{|x-y|^{2}}{t-\tau}\right) \\
& \quad \leq C_{\alpha}^{\prime} \frac{\delta(y)^{1-\alpha}}{(t-\tau)^{n+2-\alpha / 2}} \exp \left(-C^{\prime} \frac{|x-y|^{2}}{t-\tau}\right) .
\end{aligned}
$$

Also from the proof of Lemma 2.4.9,

$$
\begin{aligned}
\left|L G_{\eta}(x, t ; \eta, \lambda)\right| \leq & C \frac{|x-\eta|}{(t-\lambda)^{n+2 / 2}} \exp \left(-C \frac{|x-\eta|^{2}}{t-\lambda}\right) \\
& +C \frac{1}{(t-\lambda)^{n+1 / 2}} \exp \left(-C \frac{|x-\eta|^{2}}{t-\lambda}\right) \\
\leq & C^{\prime} \frac{1}{(t-\lambda)^{n+1 / 2}} \exp \left(-C \frac{|x-\eta|^{2}}{t-\lambda}\right) .
\end{aligned}
$$

Therefore

$$
\begin{aligned}
\left|\left(L G_{y}\right)_{2}(x, t ; y, \tau)\right| \leq C_{\alpha} \int_{\tau}^{t} d \lambda \int_{D^{\prime}} \frac{1}{(t-\lambda)^{n+1 / 2}} \exp \left(-C \frac{|x-\eta|^{2}}{t-\lambda}\right) \\
\cdot \frac{\delta(y)^{1-\alpha}}{(\lambda-\tau)^{n+2-\alpha / 2}} \exp \left(-C \frac{|\eta-y|^{2}}{\lambda-\tau}\right) d \eta \\
=C_{\alpha} \frac{\delta(y)^{1-\alpha}}{(t-\tau)^{n+1-\alpha / 2}} \exp \left(-C \frac{|x-y|^{2}}{t-\tau}\right) \frac{\Gamma(1 / 2) \Gamma(\alpha / 2)}{\Gamma(\alpha+1 / 2)}
\end{aligned}
$$

by Lemma 2.4.5. And in general

$$
\begin{aligned}
& \left|\left(L G_{y}\right)_{\nu+1}(x, t ; y, \tau)\right| \\
& \quad \leq C_{\alpha} \frac{\Gamma(1 / 2)^{\nu} \Gamma(\alpha / 2)}{\Gamma(\alpha+\nu / 2)} \frac{\delta(y)^{1-\alpha}}{(t-\tau)^{n+2-\nu-\alpha / 2}} \exp \left(-C \frac{|x-y|^{2}}{t-\tau}\right)
\end{aligned}
$$


for all $\nu=0,1,2, \ldots$ Therefore $\sum_{\nu=0}^{\infty}\left(L G_{y}\right)_{\nu+1}(x, t ; y, \tau)$ is absolutely convergent and

$$
\begin{aligned}
|\Phi(x, t ; y, \tau)| & \leq \sum_{\nu=0}^{\infty}\left|\left(L G_{y}\right)_{\nu+1}(x, t ; y, \tau)\right| \\
& \leq C_{\alpha} \frac{\delta(y)^{1-\alpha}}{(t-\tau)^{n+2-\alpha / 2}} \exp \left(-C \frac{|x-y|^{2}}{t-\tau}\right) .
\end{aligned}
$$

Lemma 2.4.11. With the same notation as Lemma 2.4.6, then the function

$$
\begin{aligned}
E(x, t ; y, \tau) & =\widetilde{G}(x, t ; y, \tau)-G_{y}(x, t ; y, \tau) \\
& =\int_{\tau}^{t} d \lambda \int_{D^{\prime}} G_{y}(x, t ; \eta, \lambda) \Phi(\eta, \lambda: y, \tau) d \eta
\end{aligned}
$$

satisfies the following inequality

$$
\left|\nabla_{x} E(x, t ; y, \tau)\right| \leq C_{\alpha} \frac{\delta(y)^{1-\alpha}}{(t-\tau)^{n+1-\alpha / 2}} \exp \left(-C \frac{|x-y|^{2}}{t-\tau}\right), \quad 0<\alpha<1,
$$

where $C_{\alpha}$ is a constant independent of $x, y \in D^{\prime}$ and $t, \tau,-1<\tau<t<1$.

Proof. By Lemma 2.4.4,

$$
\left|\nabla_{x} G_{\eta}(x, t ; y, \tau)\right| \leq \frac{C}{(t-\tau)^{n+1 / 2}} \exp \left(-C \frac{|x-y|^{2}}{t-\tau}\right)
$$

So

$$
\begin{gathered}
\left|\nabla_{x} E(x, t ; y, \tau)\right| \leq \int_{\tau}^{t} d \lambda \int_{D^{\prime}}\left|\nabla_{x} G_{y}(x, t ; \eta, \lambda)\right||\Phi(\eta, \lambda: y, \tau)| d \eta \\
\leq C_{\alpha}^{\prime} \int_{\tau}^{t} d \lambda \int_{D^{\prime}} \frac{1}{(t-\lambda)^{n+1 / 2}} \exp \left(-C \frac{|x-\eta|^{2}}{t-\lambda}\right) \\
\cdot \frac{\delta(y)^{1-\alpha}}{(\lambda-\tau)^{n+2-\alpha / 2}} \exp \left(-C \frac{|\eta-y|^{2}}{\lambda-\tau}\right) d \eta \\
\leq C_{\alpha}^{\prime} \frac{\delta(y)^{1-\alpha}}{(t-\tau)^{n+1-\alpha / 2}} \exp \left(-C \frac{|x-y|^{2}}{t-\tau}\right)
\end{gathered}
$$

by Lemma 2.4.5 and Lemma 2.4.10.

Lemma 2.4.12. With the same notation as Lemma 2.4.6,

$$
\lim _{(y, \tau) \in \bar{\Gamma}_{\beta}(0)} \int_{\partial D^{\prime}}\left|\nabla_{x} E(x, 0 ; y, \tau)\right| d \sigma(x)=0 .
$$

Proof. Choose $0<\alpha<1 / 2$ in Lemma 2.4.11. Then for $(y, \tau) \in \bar{\Gamma}_{\beta}(0)$,

$$
\begin{aligned}
\int_{\partial D^{\prime}} & \left|\nabla_{x} E(x, 0 ; y, \tau)\right| d \sigma(x) \\
& \leq C_{\alpha} \frac{\delta(y)^{1-\alpha}}{(-\tau)^{n+1-\alpha / 2}} \int_{\partial D^{\prime}} \exp \left(-C \frac{|x-y|^{2}}{-\tau}\right) d \sigma(x) \\
& \leq C_{\alpha}^{\prime} \frac{(-\tau)^{3(1-\alpha) / 2}}{(-\tau)^{n+1-\alpha / 2}} \int_{\partial D^{\prime}} \exp \left(-C^{\prime} \frac{|x|^{2}}{-\tau}\right) d \sigma(x) \\
& \leq C_{\alpha}(-\tau)^{(1-2 \alpha) / 2} \rightarrow 0 \text { as } \tau \rightarrow 0
\end{aligned}
$$


Lemma 2.4.13. The function

$$
\sum_{i, j} a_{i j}\left(y^{\prime}\right) n_{i}(x) \frac{\partial G_{y}}{\partial x_{j}}(x, t ; y, \tau), \quad x \in \partial D^{\prime}, y=\left(y^{\prime}, y_{n}^{\prime}\right) \in D^{\prime},
$$

where $-1<\tau<t<1$ and $\left(n_{i}(x)\right)_{i=1}^{n}=N_{x}$ is the inward normal to $\partial D^{\prime}$ at $x$, satisfies the following integral equation:

$$
\begin{aligned}
& \sum_{i, j} a_{i j}\left(y^{\prime}\right) n_{i}(x) \frac{\partial G_{y}}{\partial x_{j}}(x, t ; y, \tau) \\
& \quad=2 H_{y}(x, t ; y, \tau)-2 \int_{\tau}^{t} d \lambda \int_{\eta \in \partial D^{\prime}} H_{y}(x, t ; \eta, \lambda) w_{y}(\eta, \lambda ; y, \tau) d \sigma(\eta)
\end{aligned}
$$

where

$$
\begin{aligned}
w_{y}(\eta, \lambda ; y, \tau)= & \sum a_{i j}\left(y^{\prime}\right) n_{i}(\eta) \frac{\partial G_{y}}{\partial \eta_{j}}(\eta, \lambda ; y, \tau) \\
H_{y}(x, t ; \eta, \lambda)= & \sum a_{i j}\left(y^{\prime}\right) n_{i}(x) \frac{\partial Z_{y}}{\partial x_{j}}(x, t ; \eta, \lambda) \\
Z_{y}(x, t ; \eta, \lambda)= & \frac{1}{\left(\operatorname{det}\left(a^{i j}(y)\right)\right)^{1 / 2}} \\
& \cdot \frac{1}{(4 \pi(t-\lambda))^{n / 2}} \exp \left\{-\frac{\sum a^{i j}(y)\left(x_{i}-\eta_{i}\right)\left(x_{j}-\eta_{j}\right)}{4(t-\tau)}\right\}, \\
& \left(a^{i j}(y)\right)=A^{-1}=\left(a_{i j}(y)\right)^{-1}
\end{aligned}
$$

Also

$$
\int_{\tau}^{t} \int_{\partial D^{\prime}}\left|w_{y}(\eta, \lambda ; y, \tau)\right| d \sigma(\eta) \leq C<\infty, \quad C \text { independent of } y \in D^{\prime}
$$

Proof. We first observe that $Z_{y}(x, t ; \eta, \lambda)$ is the fundamental solution of

$$
L^{y} u=\sum a_{i j}(y) \frac{\partial^{2} u}{\partial x_{i} \partial x_{j}}(x, t)-\frac{\partial u}{\partial t} u(x, t)=0 .
$$

By the theory of layer potentials [LSU, p. 409] and [PO] the Green's function $G_{y}(x, t ; z, \tau)$ for the equation $L^{y}=0$ in $D^{\prime} \times(-\infty, \infty)$ is then given by

$$
G_{y}(x, t ; z, \tau)=Z_{y}(x, t ; z, \tau)-g_{y}(x, t ; z, \tau)
$$

where

$$
g_{y}(x, t ; z, \tau)=\int_{\tau}^{t} d \lambda \int_{\partial D^{\prime}} Z_{y}(x, t ; \eta, \lambda) w_{y}(\eta, \lambda ; z, \tau) d \sigma(\eta)
$$

for all $x \in R^{n}, z \in D, \tau<t$, with the density $w_{y}$ being determined from the integral equation

$$
\begin{aligned}
w_{y}(x, t ; z, \tau)= & 2 H_{y}(x, t ; z, \tau) \\
& -2 \int_{\tau}^{t} d \lambda \int_{\partial D^{\prime}} H_{y}(x, t ; \eta, \lambda) w_{y}(\eta, \lambda ; z, \tau) d \sigma(\eta) .
\end{aligned}
$$


Now for $x \in \partial D^{\prime}$,

$$
\begin{gathered}
\lim _{\substack{\bar{x}\left(\in \overline{\bar{D}^{\prime c}}\right) \rightarrow x \\
\bar{x}-x \perp N_{x}}} \sum a_{i j}(y) n_{i}(\bar{x}) \frac{\partial g_{y}}{\partial x_{j}}(\bar{x}, t ; z, \tau)=H_{y}(x, t ; z, \tau) \\
=W_{y}(x, t ; z, \tau)+\frac{1}{2} w_{y}(x, t ; z, \tau)
\end{gathered}
$$

where

$$
W_{y}(x, t ; z, \tau)=\int_{\tau}^{t} d \lambda \int_{\partial D^{\prime}} H_{y}(x, t ; \eta, \lambda) w_{y}(\eta, \lambda ; z, \tau) d \sigma(\eta)
$$

and

$$
\begin{aligned}
\lim _{\substack{\bar{x}\left(\in \overline{D^{\prime}}\right) \rightarrow x \\
\bar{x} \perp N_{x}}} \sum a_{i j}(y) n_{i}(\bar{x}) \frac{\partial g_{y}}{\partial x_{j}}(\bar{x}, t ; z, \tau)=W_{y}(x, t ; z, \tau)-\frac{1}{2} w_{y}(x, t ; z, \tau) \\
=H_{y}(x, t ; z, \tau)-w_{y}(x, t ; z, \tau) .
\end{aligned}
$$

Therefore $\forall x \in \partial D^{\prime}$,

$$
\begin{aligned}
& \sum a_{i j}(y) n_{i}(x) \frac{\partial G_{y}}{\partial x_{j}}(x, t ; z, \tau) \\
& \quad=\lim _{\substack{x\left(\in D^{\prime}\right) \rightarrow x \\
\bar{x}-x \perp N_{x}}} \sum a_{i j}(y) n_{i}(\bar{x}) \frac{\partial G_{y}}{\partial x_{j}}(\bar{x}, t ; z, \tau) \\
& =\lim _{\substack{\bar{x}\left(\in D^{\prime}\right) \rightarrow x \\
\bar{x}-x \perp N_{x}}}\left\{\sum a_{i j}(y) n_{i}(\bar{x})\left(\frac{\partial Z_{y}}{\partial x_{j}}(\bar{x}, t ; z, \tau)-\frac{\partial g_{y}}{\partial x_{j}}(\bar{x}, t ; z, \tau)\right)\right\} \\
& =\sum a_{i j}(y) n_{i}(x) \frac{\partial Z_{y}}{\partial x_{j}}(x, t ; z, \tau)-\left(H_{y}(x, t ; z, \tau)-w_{y}(x, t ; z, \tau)\right) \\
& =w_{y}(x, t ; z, \tau) .
\end{aligned}
$$

Also by the result of [LSU, p. 411], $\int_{\tau}^{t} d \lambda \int_{\partial D^{\prime}}\left|w_{y}(\eta, \lambda ; y, \tau)\right| d \sigma(\eta) \leq C<\infty$ for some constant $C$ independent of $y$.

Lemma 2.4.14. Use the same notation as Lemma 2.4.6. For any $0<2 \varepsilon \leq r_{0}$ ( $r_{0}$ as in the beginning of subsection 2.2),

$$
2 \int_{\substack{|x| \leq \varepsilon \\ x \in \partial D^{\prime}}} H_{y}(x, 0 ; y, \tau) d \sigma(x) \rightarrow \frac{\beta}{\sqrt{4 \pi}} \text { as }(y, \tau) \in \overline{\Gamma_{\beta}}(0) \rightarrow(0,0) .
$$

Proof. For $x \in \partial D^{\prime},|x| \leq \varepsilon,(y, \tau) \in \overline{\Gamma_{\beta}}(0),|y| \leq \varepsilon$,

$$
\begin{aligned}
& \sum a_{i j}(y) n_{i}(x) \frac{\partial Z_{y}}{\partial x_{j}}(x, 0 ; y, \tau) \\
& \quad=\sum_{i, j, k} a_{i j}(y) n_{i}(x)\left(-\frac{a^{j k}(y)\left(x_{k}-y_{k}\right)}{2(-\tau)}\right) Z_{y}(x, 0 ; y, \tau) \\
& \quad=\frac{-\left\langle x-y, N_{x}\right\rangle}{2(-\tau)} Z_{y}(x, 0 ; y, \tau) \\
& \quad=\frac{y_{n}^{\prime}}{2(-\tau)} Z_{y}(x, 0 ; y, \tau) .
\end{aligned}
$$


Therefore

$$
\begin{aligned}
& 2 \int_{\substack{|x| \leq \varepsilon \\
x \in \partial D^{\prime}}} H_{y}(x, 0 ; y, \tau) d \sigma(x) \\
& =\frac{y_{n}-\phi\left(y^{\prime}\right)}{\sqrt{4 \pi}(-\tau)^{3 / 2}} \cdot \frac{1}{\left(\operatorname{det} a^{i j}(y)\right)^{1 / 2}(4 \pi(-\tau))^{n-1 / 2}} \\
& \cdot \int_{\left|x^{\prime}\right| \leq \varepsilon} \exp \left(-\frac{\sum a^{i j}(y)\left(x_{i}-y_{i}\right)\left(x_{j}-y_{j}\right)}{4(-\tau)}\right) d x^{\prime} \\
& =\frac{y_{n}-\phi\left(y^{\prime}\right)}{\sqrt{4 \pi}(-\tau)^{3 / 2}}\left(\frac{1}{\left(\operatorname{det} a^{i j}(y)\right)^{1 / 2}}-1\right) \\
& \cdot \frac{1}{(4 \pi(-\tau))^{n-1 / 2}} \int_{\left|x^{\prime}\right| \leq \varepsilon} \exp \left(-\frac{\sum a^{i j}(y)\left(x_{i}-y_{i}\right)\left(x_{j}-y_{j}\right)}{4(-\tau)}\right) d x^{\prime} \\
& +\frac{y_{n}-\phi\left(y^{\prime}\right)}{\sqrt{4 \pi}(-\tau)^{3 / 2}} \cdot \frac{1}{(4 \pi(-\tau))^{n-1 / 2}} \\
& \cdot \int_{\left|x^{\prime}\right| \leq \varepsilon} \exp \left(-\frac{\sum a^{i j}(y)\left(x_{i}-y_{i}\right)\left(x_{j}-y_{j}\right)}{4(-\tau)}\right) d x^{\prime} \\
& =I_{1}+I_{2} \text {. } \\
& \left|I_{1}\right| \leq \frac{\left|y_{n}\right|+\left|\phi\left(y^{\prime}\right)\right|}{\sqrt{4 \pi}(-\tau)^{3 / 2}}\left|\frac{1}{\left(\operatorname{det} a^{i j}(y)\right)^{1 / 2}}-1\right| \\
& \cdot \frac{1}{(4 \pi(-\tau))^{n-1 / 2}} \int_{\substack{\left|x^{\prime}\right| \leq \varepsilon \\
x^{\prime} \in R^{n-1}}} \exp \left(-C \frac{|x-y|^{2}}{(-\tau)}\right) d x^{\prime} \\
& \leq C\left|\frac{1}{\left(\operatorname{det}\left(a^{i j}\left(y^{\prime}\right)\right)\right)^{1 / 2}}-1\right| \\
& \frac{1}{(4 \pi(-\tau))^{n-1 / 2}} \int_{\substack{\left|x^{\prime}\right| \leq \varepsilon \\
x^{\prime} \in R^{n-1}}} \exp \left(-C^{\prime} \frac{\left|x^{\prime}\right|^{2}}{(-\tau)}\right) d x^{\prime} \\
& \leq C^{\prime}\left|\frac{1}{\left(\operatorname{det}\left(a^{i j}\left(y^{\prime}\right)\right)\right)^{1 / 2}}-1\right| \rightarrow 0 \quad \text { as }(y, \tau) \in \bar{\Gamma}_{\beta}(0) \rightarrow(0,0)
\end{aligned}
$$

since $\left(a^{i j}(y)\right)^{-1} \rightarrow I=$ identity matrix as $y \rightarrow 0$.

For $I_{2}$, since $\left|y^{\prime}\right| \leq C(-\tau)^{3 / 2}$, we have

$$
\frac{y_{n}-\phi\left(y^{\prime}\right)}{(-\tau)^{3 / 2}}=\frac{y_{n}}{(-\tau)^{3 / 2}}+\frac{O\left(y^{\prime 2}\right)}{(-\tau)^{3 / 2}}=\beta+O\left(\tau^{3 / 2}\right) \rightarrow \beta \quad \text { as } \tau \rightarrow 0
$$

and

$$
\begin{gathered}
\frac{1}{(4 \pi(-\tau))^{n-1 / 2}} \int_{\substack{\left|x^{\prime}\right| \leq \varepsilon \\
x^{\prime} \in R^{n-1}}} \exp \left(-\frac{\sum a^{i j}(y)\left(x_{i}-y_{i}\right)\left(x_{j}-y_{j}\right)}{4(-\tau)}\right) d x^{\prime} \\
\rightarrow \frac{1}{(4 \pi)^{n-1 / 2}} \int_{\substack{\bar{x}^{\prime} \in R^{n-1}\\
}} \exp \left(-\left|\bar{x}^{\prime}\right|^{2} / 4\right) d \bar{x}^{\prime}=1 \quad \text { as } \tau \rightarrow 0
\end{gathered}
$$


Therefore $I_{2} \rightarrow \beta / \sqrt{4 \pi}$ as $(y, \tau) \in \bar{\Gamma}_{\beta}(0) \rightarrow(0,0)$. So $2 \int_{\substack{|x| \leq \varepsilon \\ x \in \partial D^{\prime}}} H_{y}(x, 0 ; y, \tau) d \sigma(x)=I_{1}+I_{2} \rightarrow \frac{\beta}{\sqrt{4 \pi}}$ as $(y, \tau) \in \bar{\Gamma}_{\beta}(0) \rightarrow(0,0)$.

Lemma 2.4.15. With the same notation as Lemma 2.4.13, we have

$$
\begin{aligned}
& \int_{x^{\prime} \in R^{n-1}} \sum a_{i j}(y) n_{i}(x) \frac{\partial G_{y}}{\partial x_{j}}(x, 0 ; y, \tau) d x^{\prime} \\
& \quad=\int_{\substack{|x| \leq \varepsilon \\
x^{\prime} \in \bar{\partial} D^{\prime}}} \sum a_{i j}(y) n_{i}(x) \frac{\partial G_{y}}{\partial x_{j}}(x, 0 ; y, \tau) d \sigma(x) \\
& \quad \rightarrow \frac{\beta}{\sqrt{4 \pi}} \text { as }(y, \tau) \in \bar{\Gamma}_{\beta}(0) \rightarrow(0 ; 0) .
\end{aligned}
$$

Proof. By Lemma 2.4.13,

$$
\begin{aligned}
& \sum a_{i j}(y) n_{i}(x) \frac{\partial G_{y}}{\partial x_{j}}(x, 0 ; y, \tau)=2 H_{y}(x, 0 ; y, \tau) \\
& \quad-2 \int_{\tau}^{0} d \lambda \int_{\eta \in \partial D^{\prime}} H_{y}(x, 0 ; \eta, \lambda) w_{y}(\eta, \lambda ; y, \tau) d \sigma(\eta) .
\end{aligned}
$$

Also $x_{n}^{\prime}=0$ for $x \in \partial D^{\prime},|x| \leq \varepsilon$, therefore

$$
\begin{aligned}
\int_{\substack{|x| \leq \varepsilon \\
x^{\prime} \in \partial D^{\prime}}} & \sum a_{i j}(y) n_{i}(x) \frac{\partial G_{y}}{\partial x_{j}}(x, 0 ; y, \tau) d \sigma(x) \\
= & \int_{\substack{|x| \leq \varepsilon \\
x^{\prime} \in \partial D^{\prime}}} \sum a_{i j}(y) n_{i}(x) \frac{\partial G_{y}}{\partial x_{j}}(x, 0 ; y, \tau) d x^{\prime} \\
= & \int_{\substack{|x| \leq \varepsilon \\
x^{\prime} \in \partial D^{\prime}}}(-2) \int_{\tau}^{0} d \lambda\left\{\int_{\substack{|\eta| \leq 2 \varepsilon \\
\eta \in \partial D^{\prime}}}+\int_{\substack{|\eta|>2 \varepsilon \\
\eta \in \partial D^{\prime}}}\right\} \\
& +2 \int_{\substack{|x| \leq \varepsilon \\
x^{\prime} \in \partial D^{\prime}}} H_{y}(x, 0 ; y, \tau) d x^{\prime} \\
= & J_{1}+J_{2}+J_{3} .
\end{aligned}
$$

Now for $x, \eta \in \partial D^{\prime},|x|,|\eta| \leq 2 \varepsilon$, we have $x_{n}^{\prime}=\eta_{n}^{\prime}=0$ and $N_{x}=$ $(0, \ldots, 0,1)$. So

$$
\begin{aligned}
H_{y}(x, 0 ; \eta, \lambda) & =\sum a_{i j}(y) n_{i}(x) \frac{\partial Z_{y}}{\partial x_{j}}(x, 0 ; \eta, \lambda) \\
& =\frac{-\left\langle N_{x}, x-\eta\right\rangle}{2(-\lambda)} Z_{y}(x, 0 ; \eta, \lambda) \\
& =0 .
\end{aligned}
$$


Therefore $J_{1}=0$. For $|x| \leq \varepsilon,|\eta| \geq 2 \varepsilon, x, \eta \in \partial D^{\prime},(y, \tau) \in \bar{\Gamma}_{\beta}(0)$, $-1<\tau<\lambda<0$, since

$$
\begin{aligned}
\left|H_{y}(x, 0 ; \eta, \lambda)\right| & =\left|\sum a_{i j}(y) n_{i}(x) \frac{\partial Z_{y}}{\partial x_{j}}(x, 0 ; \eta, \lambda)\right| \\
& =\left|\frac{-\left\langle N_{x}, x-\eta\right\rangle}{2(-\lambda)}\right|\left|Z_{y}(x, 0 ; \eta, \lambda)\right| \\
& \leq C \frac{1}{(-\lambda)^{n+1 / 2}} \exp \left(-C \frac{\varepsilon^{2}}{(-\lambda)}\right) .
\end{aligned}
$$

Therefore $\left|J_{2}\right|$ is

$$
\begin{aligned}
& \leq C \int_{\left|x^{\prime}\right| \leq \varepsilon} \int_{\tau}^{0} d \lambda \int_{\substack{\eta \in \partial D^{\prime} \\
|\eta| \geq 2 \varepsilon}} \frac{1}{(-\lambda)^{n+1 / 2}} \exp \left(-C \frac{\varepsilon^{2}}{(-\lambda)}\right)|w(\eta, \lambda, y, \tau)| d \sigma(\eta) d x^{\prime} \\
& \leq C \varepsilon^{n-1} \sup _{\tau \leq \lambda \leq 0} \frac{C}{|\lambda|^{n-1 / 2}} \exp \left(-C \frac{\varepsilon^{2}}{|\lambda|}\right) \rightarrow 0 \text { as }(y, \tau)\left(\in \bar{\Gamma}_{\beta}(0)\right) \rightarrow(0,0) .
\end{aligned}
$$

Also by Lemma $2.4 .14, J_{3} \rightarrow \beta / \sqrt{4 \pi}$ as $(y, \tau)\left(\in \bar{\Gamma}_{\beta}(0)\right) \rightarrow(0,0)$. Therefore

$$
\lim _{(y, \tau) \in \bar{\Gamma}_{\beta}(0)} \int_{\substack{\tau \rightarrow 0 \\ x^{\prime} \in R^{\prime} \mid \leq \varepsilon}} \sum a_{i j}\left(y^{\prime}\right) n_{i}(x) \frac{\partial G_{y}}{\partial x_{j}}(x, 0 ; y, \tau) d x^{\prime}=\frac{\beta}{\sqrt{4 \pi}}
$$

Lemma 2.4.16. With the same notation as Lemma 2.4.6 and Lemma 2.4.13, we have

$$
\lim _{\substack{(y, \tau) \in \bar{\Gamma}_{\beta}(0) \\ \tau \rightarrow 0}} \int_{\substack{\left|x^{\prime}\right| \leq \varepsilon \\ x^{\prime} \in R^{n-1}}} \frac{\partial \widetilde{G}}{\partial x_{n}^{\prime}}(x, 0 ; y, \tau) d x^{\prime}=\frac{\beta}{\sqrt{4 \pi}} .
$$

Proof. By Lemma 2.4.6,

$$
\begin{aligned}
& \int_{\substack{\left|x^{\prime}\right| \leq \varepsilon \\
x^{\prime} \in R^{n-1}}} \frac{\partial \widetilde{G}}{\partial x_{n}^{\prime}}(x, 0 ; y, \tau) d x^{\prime} \\
& =\int_{\substack{\left|x^{\prime}\right| \leq \varepsilon \\
x^{\prime} \in R^{n-1}}} \frac{\partial G_{y}}{\partial x_{n}^{\prime}}(x, 0 ; y, \tau) d x^{\prime} \\
& \quad+\int_{\substack{\left|x^{\prime}\right| \leq \varepsilon \\
x^{\prime} \in R^{n-1}}} \frac{\partial E}{\partial x_{n}^{\prime}}(x, 0 ; y, \tau) d x^{\prime} \\
& =I_{1}+I_{2} .
\end{aligned}
$$


By Lemma 2.4.12, $I_{2} \rightarrow 0$ as $(y, \tau)\left(\in \bar{\Gamma}_{\beta}(0)\right) \rightarrow(0,0)$. Also

$$
\begin{aligned}
\left|I_{1}-\frac{\beta}{\sqrt{4 \pi}}\right| \leq & \left|I_{1}-\int_{\substack{\left|x^{\prime}\right| \leq \varepsilon \\
x^{\prime} \in R^{n-1}}} \sum a_{i j}\left(y^{\prime}\right) n_{i}(x) \frac{\partial G_{y}}{\partial x_{j}}(x, 0 ; y, \tau) d x^{\prime}\right| \\
& +\left|\int_{\substack{\left|x^{\prime}\right| \leq \varepsilon \\
x^{\prime} \in R^{n-1}}} \sum a_{i j}\left(y^{\prime}\right) n_{i}(x) \frac{\partial G_{y}}{\partial x_{j}}(x, 0 ; y, \tau) d x^{\prime}-\frac{\beta}{\sqrt{4 \pi}}\right| \\
= & E_{1}+E_{2} .
\end{aligned}
$$

By Lemma 2.4.15, $E_{2} \rightarrow 0$ as $(y, \tau)\left(\epsilon \bar{\Gamma}_{\beta}(0)\right) \rightarrow(0,0)$. While

$$
\begin{aligned}
E_{1}= & \left|I_{1}-\int_{\substack{\left|x^{\prime}\right| \leq \varepsilon \\
x^{\prime} \in R^{n-1}}} \sum a_{n j}\left(y^{\prime}\right) \frac{\partial G_{y}}{\partial x_{j}}(x, 0 ; y, \tau) d x^{\prime}\right| \quad\left(n_{i}(x)\right)_{i=1}^{n}=(0, \ldots, 0,1) \\
\leq & C\left\{\sum_{1 \leq j \leq n-1}\left|a_{n j}\left(y^{\prime}\right)\right|+\left|a_{n n}\left(y^{\prime}\right)-1\right|\right\} \int_{\substack{\left|x^{\prime}\right| \leq \varepsilon \\
x^{\prime} \in R^{n-1}}} \max _{j}\left|\frac{\partial G_{y}}{\partial x_{j}}(x, 0 ; y, \tau)\right| d x^{\prime} \\
\leq & C\left\{\sum_{1 \leq j \leq n-1}\left|\frac{\partial \phi}{\partial y_{j}}\left(y^{\prime}\right)\right|+\left|\nabla_{y^{\prime}} \phi\left(y^{\prime}\right)\right|^{2}\right\} \\
& \cdot \int_{\left|x^{\prime}\right| \leq \varepsilon} \frac{\delta(y)}{|\tau|^{n+2 / 2}} \exp \left(-C \frac{|x-y|^{2}}{|\tau|}\right) d x^{\prime} \\
\leq & C^{\prime}\left\{\sum_{1 \leq j \leq n-1}\left|\frac{\partial \phi}{\partial y_{j}}\left(y^{\prime}\right)\right|+\left|\nabla_{y^{\prime}} \phi\left(y^{\prime}\right)\right|^{2}\right\} \\
& \cdot \frac{1}{|\tau|^{n-1 / 2}} \int_{\substack{\left|x^{\prime}\right| \leq \varepsilon \\
x^{\prime} \in R^{n-1}}} \exp \left(-C^{\prime} \frac{\left|x^{\prime}\right|^{2}}{|\tau|}\right) d x^{\prime} \\
\leq & C^{\prime}\left\{\sum_{1 \leq j \leq n-1}\left|\frac{\partial \phi}{\partial y_{j}}\left(y^{\prime}\right)\right|+\left|\nabla_{y^{\prime}} \phi\left(y^{\prime}\right)\right|^{2}\right\} \rightarrow 0
\end{aligned}
$$

as $(y, \tau)\left(\in \bar{\Gamma}_{\beta}(0)\right) \rightarrow(0,0)$ since $\nabla \phi(0)=0$. Hence $\left|I_{1}-\beta / \sqrt{4 \pi}\right| \rightarrow 0$ as $(y, \tau) \quad\left(\in \bar{\Gamma}_{\beta}(0)\right) \rightarrow(0,0)$. So

$$
\lim _{\substack{(y, \tau) \in \bar{\Gamma}_{\beta}(0) \\ \tau \rightarrow 0}} \int_{\substack{\left|x^{\prime}\right| \leq \varepsilon \\ x^{\prime} \in R^{n-1}}} \frac{\partial \widetilde{G}}{\partial x_{n}^{\prime}}(x, 0 ; y, \tau) d x^{\prime}=\frac{\beta}{\sqrt{4 \pi}} .
$$

We are now ready for the proof of Theorem 2.4.1. 
Proof of Theorem 2.4.1.

$$
\begin{aligned}
\mid \int_{x \in \partial D} & \frac{\partial G}{\partial N_{x}}(x, 0 ; y, \tau) d \sigma(x)-\frac{\beta}{\sqrt{4 \pi}} \mid \\
\leq & \int_{\substack{x \in \partial D \\
\left|x^{\prime}\right| \geq \varepsilon}}\left|\frac{\partial G}{\partial N_{x}}(x, 0 ; y, \tau)\right| d \sigma(x) \\
& +\left|\int_{\substack{x \in \partial D \\
\left|x^{\prime}\right| \leq \varepsilon}} \nabla_{x} G(x, 0 ; y, \tau) \cdot\left(-\nabla \phi\left(x^{\prime}\right), 1\right) d x^{\prime}-\frac{\beta}{\sqrt{4 \pi}}\right| \\
\leq & \int_{\substack{x \in \partial D \\
\left|x^{\prime}\right| \geq \varepsilon}}\left|\frac{\partial G}{\partial N_{x}}(x, 0 ; y, \tau)\right| d \sigma(x) \\
& +\sup _{\left|x^{\prime}\right| \leq \varepsilon}\left|\nabla \phi\left(x^{\prime}\right)\right| \cdot \int_{x \in \partial D}|\nabla x G(x, 0 ; y, \tau)| d x^{\prime} \\
& +\left|\int_{\substack{\left|x^{\prime}\right| \leq \varepsilon \\
x^{\prime} \mid \leq \varepsilon}} \frac{\partial G}{\partial x_{n}^{\prime}}(x, 0 ; y, \tau) d x^{\prime}-\frac{\beta}{\sqrt{4 \pi}}\right| \\
= & I_{1}^{\prime}+I_{2}^{\prime}+I_{3}^{\prime}
\end{aligned}
$$

since

$$
\frac{\partial G}{\partial x_{n}}(x, 0 ; y, \tau)=\frac{\partial \widetilde{G}}{\partial x_{n}^{\prime}}(x, 0 ; y, \tau) \text { and } \quad\left|\frac{\partial\left(x^{\prime}, x_{n}\right)}{\partial\left(x^{\prime}, x_{n}^{\prime}\right)}\right|=1
$$

with $x_{n}^{\prime}=x_{n}-\phi\left(x^{\prime}\right)$. Now

$$
\begin{aligned}
\int_{\substack{x \in \partial D^{\prime} \\
\left|x^{\prime}\right| \leq \varepsilon}}\left|\nabla_{x} G(x, 0 ; y, \tau)\right| d x^{\prime} & \leq \int_{\substack{x \in \partial D^{\prime} \\
\left|x^{\prime}\right| \leq \varepsilon}} \frac{\delta(y)}{|\tau|^{n+2 / 2}} \exp \left(-C \frac{|x-y|^{2}}{|\tau|}\right) d x^{\prime} \\
& \leq \int_{\substack{x \in \partial D^{\prime} \\
\left|x^{\prime}\right| \leq \varepsilon}} \frac{1}{|\tau|^{n-1 / 2}} \exp \left(-C \frac{\left|x^{\prime}\right|^{2}}{|\tau|}\right) d x^{\prime} \leq C^{\prime}
\end{aligned}
$$

for $(y, \tau) \in \bar{\Gamma}_{\beta}(0)$.

Therefore $\left|I_{2}^{\prime}\right| \leq C^{\prime \prime} \sup _{\left|x^{\prime}\right| \leq \varepsilon}\left|\nabla^{2} \phi\left(x^{\prime}\right)\right|\left|x^{\prime}\right| \leq C^{\prime \prime \prime} \varepsilon$.

By Lemma 2.4.16, $I_{3}^{\prime} \rightarrow 0$ as $(y, \tau) \in \bar{\Gamma}_{\beta}(0) \rightarrow 0$. Also $I_{1}^{\prime} \rightarrow 0$ as $\tau \rightarrow 0$. Therefore

$$
\begin{aligned}
& \varlimsup_{\substack{(y, \tau) \in \bar{\Gamma}_{\beta}(0) \\
\tau \rightarrow 0}}\left|\int_{x \in \partial D} \frac{\partial G}{\partial N_{x}}(x, 0 ; y, \tau) d \sigma(x)-\frac{\beta}{\sqrt{4 \pi}}\right| \leq C \varepsilon \quad \forall \varepsilon>0 \\
& \Rightarrow \lim _{\substack{(y, \tau) \in \bar{\Gamma}_{\beta}(0) \\
\tau \rightarrow 0}} \int_{x \in \partial D} \frac{\partial G}{\partial N_{x}}(x, 0 ; y, \tau) d \sigma(x)=\frac{\beta}{\sqrt{4 \pi}} .
\end{aligned}
$$

2.5. An example. In this section we will give an example of a solution of the heat equation in $R^{2}$ to show that the index $3 / 2$ on $t$ in the definition of the space-time cone for the corner points of a cylinder is essentially sharp. 
By the result of P. Hartman and A. Wintner [HW] and Hattemer [H], if

$$
\theta(x, t)=\sum_{k=-\infty}^{\infty}(4 \pi t)^{-1 / 2} \exp \left(-\frac{(x+2 k)^{2}}{4 t}\right)
$$

then the Green function for the heat equation in the region $D_{1} \times(0, \infty)$, where $D_{1}=[0,1]^{2}$, is given by

$$
G\left(\left(x_{1}, x_{2}\right), t ;\left(y_{1}, y_{2}\right), \tau\right)=\prod_{i=1}^{2}\left\{\theta\left(x_{i}-y_{i}, t-\tau\right)-\theta\left(x_{i}+y_{i}, t-\tau\right)\right\}
$$

where $0 \leq s<t<\infty$. Therefore

$$
\left.\frac{\partial G}{\partial N_{\left(y_{1}, y_{2}\right)}}\right|_{y_{1}=0}=-2 \theta_{x_{1}}\left(x_{1}, t-\tau\right) \cdot\left\{\theta\left(x_{2}-y_{2}, t-\tau\right)-\theta\left(x_{2}+y_{2}, t-\tau\right)\right\}
$$

where $\partial / \partial N_{\left(y_{1}, y_{2}\right)}$ is the derivative in the direction of the inward normal $N_{\left(y_{1}, y_{2}\right)}$ to $\partial D_{1}$ at $\left(y_{1}, y_{2}\right) \in \partial D_{1}$. By the representation theorem in subsection 2.2,

$$
u(x, t)=-2 \theta_{x_{1}}\left(x_{1}, t-\tau\right) \cdot \int_{0}^{1}\left\{\theta\left(x_{2}-y_{2}, t-\tau\right)-\theta\left(x_{2}+y_{2}, t-\tau\right)\right\} d y_{2}
$$

with $x=\left(x_{1}, x_{2}\right) \in D_{1}, t>0$, is the strong solution of the (IDP) in $D_{1} \times$ $(0, \infty)$ with initial trace 0 on $D_{1}$ and trace $d \lambda$ on $\partial D_{1}$ that equals $d x_{2}$ on $\{0\} \times[0,1]$ and equals 0 on $\partial D_{1} \backslash\{0\} \times[0,1]$.

Fix a point $\left(x_{1}^{0}, x_{2}^{0}\right)=\left(0, x_{2}^{0}\right) \in \partial D, 0<x_{2}^{0}<1$, and let $(x, t) \in D_{1} \times$ $(0, \infty)$. Then

$$
\int_{0}^{1}\left\{\theta\left(x_{2}-y_{2}, t-\tau\right)-\theta\left(x_{2}+y_{2}, t-\tau\right)\right\} d y_{2}
$$

is the bounded solution of the heat equation in $[0,1] \times(0, \infty)$ with initial value 1 on $(0,1) \times\{0\}$ and boundary value 0 on the lateral sides of $[0,1] \times(0, \infty)$. Therefore

$$
\int_{0}^{1}\left\{\theta\left(x_{2}-y_{2}, t-\tau\right)-\theta\left(x_{2}+y_{2}, t-\tau\right)\right\} d y_{2} \rightarrow 1 \quad \text { if }\left|x_{2}-x_{2}^{0}\right| \leq C t^{3 / 2} \rightarrow 0 .
$$

On the other hand,

$$
\begin{gathered}
-2 \theta_{x_{1}}\left(x_{1}, t-\tau\right)=2 \sum_{k=-\infty}^{\infty}(4 \pi t)^{-1 / 2} \cdot \frac{2\left(x_{1}+2 k\right)}{4 t} \exp \left(-\frac{\left(x_{1}+2 k\right)^{2}}{4 t}\right) \\
=\frac{x_{1}}{\sqrt{4 \pi} t^{3 / 2}} \exp \left(-\frac{x_{1}^{2}}{4 t}\right)+\sum_{\substack{k=-\infty \\
k \neq 0}}^{\infty} \frac{\left(x_{1}+2 k\right)}{\sqrt{4 \pi} t^{3 / 2}} \exp \left(-\frac{\left(x_{1}+2 k\right)^{2}}{4 t}\right) .
\end{gathered}
$$

If $x_{1}=\beta t^{a}$, then the second term always goes to 0 as $t \rightarrow 0$ while the first term will converge to 0 if $a>3 / 2, \infty$ if $a<3 / 2, \beta / \sqrt{4 \pi}$ if $a=3 / 2$. Hence

$$
\begin{aligned}
u(x, t) & \rightarrow 0 \text { as } t \rightarrow 0 \text { if } a>3 / 2 \text { and } x_{1}=\beta t^{a},\left|x_{2}-x_{2}^{0}\right| \leq C t^{3 / 2}, \\
& \rightarrow \infty \text { as } t \rightarrow 0 \text { if } a<3 / 2 \text { and } x_{1}=\beta t^{a},\left|x_{2}-x_{2}^{0}\right| \leq C t^{3 / 2}, \\
& \rightarrow \frac{\beta}{\sqrt{4 \pi}} \text { as } t \rightarrow 0 \text { if } a=3 / 2 \text { and } x_{1}=\beta t^{a},\left|x_{2}-x_{2}^{0}\right| \leq C t^{3 / 2}
\end{aligned}
$$


Therefore the index $3 / 2$ on $t$ in the definition of the space-time cone is essentially sharp.

\section{ACKNOWLEDGMENT}

This paper is part of the thesis [HU] I wrote under the direction of Professor Carlos E. Kenig, my thesis advisor. I wish to thank him for suggesting this problem to me and for his constant encouragement and advice during the preparation of the paper. I would also like to thank Professor Robert Fefferman and Professor Russell M. Brown for many helpful discussions of the problem.

\section{REFERENCES}

[D] E. B. Davies, Heat kernels and spectral theory, Cambridge Univ. Press, New York, 1989.

[DK1] B. E. J. Dahlberg and C. E. Kenig, Nonnegative solutions of the generalized porous medium equation, Rev. Mat. Iberoamerican 2 (1986), 267-305.

[DK2] _ Nonnegative solutions of the initial Dirichlet problem for generalized porous medium equation in cylinders, J. Amer. Math. Soc. 1 (1988), 401-412.

[F] A. Friedman, Partial differential equations of parabolic type, Krieger, Malabar, Florida, 1983.

[FGS] E. B. Fabes, N. Garofalo, and S. Salsa, A backward Harnack inequality and Fatou theorem for nonnegative solutions of parabolic equations, Illinois J. Math. 30 (1986), 536-565.

[FS] E. B. Fabes and S. Salsa, Estimate of caloric measure and the initial Dirichlet problem for the heat equation in Lipschitz cylinders, Trans. Amer. Math. Soc. 279 (1983), 635-650.

[GW] Michael Grüter and K. O. Widman, The Green function for uniformly elliptic equations, Manuscripta Math. 37 (1982), 303-342.

[H] J. R. Hattemer, Boundary behaviour of temperatures. I, Studia Math. 25 (1964), 111-155.

[HU] Kin Ming Hui, Thesis (in preparation).

[HW] Philip Hartman and A. Wintner, On the solutions of the equation of heat conduction, Amer. J. Math. 72 (1950), 367-395.

[JK] D. S. Jerison and C. E. Kenig, Boundary value problems on Lipschitz domains, Studies in Partial Differential Equations, MAA Studies in Math., 1982.

[K] J. T. Kemper, Temperatures in several variables: kernel functions, representations, and parabolic boundary values, Trans. Amer. Math. Soc. 167 (1972), 243-262.

[LSU] O. A. Ladyženskaja, V. A. Solonnikov, and N. N. Ural'ceva, Linear and quasi-linear equations of parabolic type, Transl. Math. Mono., Vol. 23, Amer. Math. Soc., Providence, R.I., 1968.

[Po] W. Pogorzelski, Étude d'une fonction de Green et du problème aux limites pour l'equation parabolique normale, Ann. Polon. Math. 4 (1958), 288-307.

[S] E. M. Stein, Singular integrals and differentiability properties of functions, Princeton Univ. Press, Princeton, N.J., 1971.

Department of Mathematics, University of Chicago, Chicago, Illinois 60637

Current address: Institute of Mathematics, Academia Sinica, Nankang, Taipei, Taiwan, R.O.C.

E-mail address: makmhui@twnas886.bitnet 